\title{
NUMERICAL ANALYSIS \\ OF THE DETERMINISTIC PARTICLE METHOD APPLIED TO THE WIGNER EQUATION
}

\author{
ANTON ARNOLD AND FRANCIS NIER
}

\begin{abstract}
The Wigner equation of quantum mechanics has the form of a kinetic equation with a pseudodifferential operator in a Fourier integral form which requires great care in the numerical approximation. This paper is concerned with the numerical analysis of the weighted particle method, introduced by S. Mas-Gallic and P. A. Raviart, applied to this equation. In particular, we will prove convergence of the method in a physically relevant case, where the Wigner equation models the quantum tunneling of electrons through a potential barrier.
\end{abstract}

\section{INTRODUCTION}

In the past few years, there has been a renewal of interest in the Wigner-or quantum Liouville-equation. This equation, first proposed by Wigner in [2], provides a kinetic model which takes into account quantum effects in transport phenomena. Such an application of this equation was suggested by solid state physicists $[3,4]$ in order to describe the behavior of electrons in ultraintegrated electronic devices. The typical example is the resonant tunneling diode whose performance is based on quantum tunneling through a potential barrier.

The simulations which rely on the Wigner model aim to determine the current-voltage characteristic of these devices as well as their behavior away from equilibrium. In this framework, the usual numerical methods are finite difference schemes $[3,4]$ and spectral methods [5].

Here we shall investigate a weighted particle method which has been used for a few years for integro-differential equations arising from the classical kinetic theory. In particular, the numerical analysis of this method was carried out in the context of the linearized Boltzmann equation $[6,7]$ and of the semiconductor Boltzmann equation [8], where the integral operator models collisions of physical particles. However, the corresponding results do not carry over to the Wigner equation because of the particular form of the Fourier integral operator which describes quantum effects due to a potential barrier. Moreover, the usual mathematical analysis of this equation $[9,18]$ relies on semigroup theory in Hilbert spaces, especially $L^{2}\left(\mathbb{R}^{n}\right)$, which is the natural framework of quantum mechanics. But this context cannot be used in the numerical analysis of the particle method, which needs $L^{p}$ and $W^{m, p}$ estimates with $p=1$ or

Received February 8, 1991.

1991 Mathematics Subject Classification. Primary 65M15, 35S05, 81 S30. 
$p=\infty[10,11]$. As we will see, the $W^{m, p}$ estimates, and therefore the order of convergence, are related to the regularity of the potential $V(x)$, which will model the potential barrier.

The numerical application of the proposed method to the Wigner equation will be the topic of a subsequent paper [19].

The outline of this paper is as follows. Section 2 introduces the Wigner equation and the particle method, $\S 3$ provides a priori estimates in $W^{m, p}$ spaces of the exact solution, and the convergence analysis is done in $\S 4$. The paper ends with a convergence proof of the particle method in a physically relevant case where the potential is not smooth. This last result, given in $\S 5$, is obtained by weak compactness arguments which are not standard in the analysis of particle methods.

\section{THE WigNer EQUATION AND THE PARTICLE METHOD}

The Wigner equation models the motion of electrons in an external electrostatic potential, which we shall decompose in order to describe quantum tunneling effects: the electrons are accelerated by a uniform electric field $E$ and partially tunnel through a potential barrier given by the real-valued function $V(x), x \in \mathbb{R}^{d} \quad(d=1,2$, or 3$)$. The Wigner equation governs the evolution of a (quasi)-distribution function $w(x, v, t)$, where $x \in \mathbb{R}^{d}, v \in \mathbb{R}^{d}$, and $t \in \mathbb{R}^{+}$are respectively the position, velocity, and time coordinates:

$$
\left\{\begin{array}{l}
\partial_{t} w+v \partial_{x} w+E \partial_{v} w=\theta\left(x, \partial_{v}\right) w, \\
w(t=0)=w_{I}
\end{array}\right.
$$

The operator $\theta\left(x, \partial_{v}\right)$ is a pseudodifferential operator related to the potential $V$. It is defined by

$$
\theta\left(x, \partial_{v}\right) w=\frac{1}{i}\left[V\left(x+\frac{i}{2} \partial_{v}\right)-V\left(x-\frac{i}{2} \partial_{v}\right)\right] w,
$$

or in an equivalent integral form

$$
\begin{gathered}
(\theta w)(x, v) \\
=(2 \pi)^{-d / 2} \int_{\mathbb{R}_{\eta}^{d}} \frac{1}{i}\left[V\left(x-\frac{\eta}{2}\right)-V\left(x+\frac{\eta}{2}\right)\right] \hat{w}(x, \eta) e^{i v \cdot \eta} d \eta, \\
\hat{w}(x, \eta)=(2 \pi)^{-d / 2} \int_{\mathbb{R}_{\xi}^{d}} w(x, \xi) e^{-i \xi \cdot \eta} d \xi .
\end{gathered}
$$

The term $E \partial_{v} w$ can be included in the pseudodifferential operator by adding $-E \cdot x$ to the potential $V(x)$. Without restricting the generality of the model, we will first assume that the function $V$ belongs to $L^{2}\left(\mathbb{R}^{d}\right) \cap L^{\infty}\left(\mathbb{R}^{d}\right)$. Then a semigroup analysis provided in [9] states that the operator $-v \partial_{x}-E \partial_{v}+\theta\left(x, \partial_{v}\right)$ generates a strongly continuous unitary group in $L^{2}\left(\mathbb{R}_{x}^{d} \times \mathbb{R}_{v}^{d}\right)$. Thus, if the initial data $w_{I}$ belongs to $L^{2}\left(\mathbb{R}^{2 d}\right)$, there exists a unique mild solution $w$ of equation (2.1) which satisfies

$$
\|w(t)\|_{L^{2}\left(\mathbb{R}^{2 d}\right)}=\left\|w_{I}\right\|_{L^{2}\left(\mathbb{R}^{2 d}\right)} .
$$

Since $w(t) \in L^{2}\left(\mathbb{R}^{2 d}\right)$ for any $t \in \mathbb{R}^{+}$, the right-hand side of equation (2.1) appears as a convolution product of two $L^{2}$-functions with respect to the velocity 
variable:

$$
\begin{aligned}
(\theta w)(x, v, t) & =\varphi(x, v) *_{v} w(x, v, t) \\
& =\int_{\mathbb{R}_{v^{\prime}}^{d}} \varphi\left(x, v-v^{\prime}\right) w\left(x, v^{\prime}, t\right) d v^{\prime} .
\end{aligned}
$$

The function $\varphi$ is the inverse Fourier transform (with respect to the velocity variable) of the function $\frac{1}{i}\left[V\left(x-\frac{1}{2} \eta\right)-V\left(x+\frac{1}{2} \eta\right)\right]$ and is defined by

$$
\begin{aligned}
\varphi(x, v) & =(2 \pi)^{-d} \int \frac{1}{i}\left[V\left(x-\frac{\eta}{2}\right)-V\left(x+\frac{\eta}{2}\right)\right] e^{i v \cdot \eta} d \eta \\
& =2\left(\frac{2}{\pi}\right)^{d / 2} \operatorname{Im}\left[e^{2 i v \cdot x} \widehat{V}(2 v)\right] .
\end{aligned}
$$

Then the Wigner equation can be written as

$$
\left\{\begin{array}{l}
\partial_{t} w+v \partial_{x} w+E \partial_{v} w=\varphi *_{v} w \\
w(t=0)=w_{I}
\end{array}\right.
$$

The right-hand side of this equation is local in position and nonlocal in velocity, like the collision operator of the Boltzmann type equations $[6,7,8]$. This suggests to set up a similar particle method as the one developed for these equations.

This numerical method relies on the definition of a discrete set of particles at positions $\left(x_{i}(t), v_{i}(t)\right), i \in \mathbb{Z}^{2 d}$, with constant phase space control volumes $\lambda_{i}$ and time-depending weights $w_{i}(t)$. These particles must not be considered as physical particles but as purely mathematical quantities. Then the solution $w$ is approximated in measure by a linear combination of delta functions:

$$
w(x, v, t) \simeq \tilde{w}(x, v, t)=\sum_{i \in \mathbb{Z}^{2 d}} \lambda_{i} w_{i}(t) \delta\left(x-x_{i}(t)\right) \otimes \delta\left(v-v_{i}(t)\right)
$$

Moreover, for a continuous Wigner function $w$, the weights $w_{i}(t)$ will turn out to be an approximation for $w\left(x_{i}(t), v_{i}(t), t\right)$.

The particles are moved along the characteristics of the left-hand side of (2.5):

$$
\frac{d x_{i}}{d t}=v_{i}, \quad \frac{d v_{i}}{d t}=E .
$$

In order to determine the time evolution of the weights, we first notice that the exact solution $w$ of equation (2.5) satisfies

$$
\frac{d}{d t} w\left(x_{i}(t), v_{i}(t), t\right)=\left(\varphi *_{v} w\right)\left(x_{i}(t), v_{i}(t), t\right)=(\theta w)\left(x_{i}(t), v_{i}(t), t\right) .
$$

Now we have to find an approximation of the right-hand integral operator, which is local in $x$. A simple argument shows that it does not make sense to directly apply $\theta$ to the particle approximation $\tilde{w}$ : When the particles are initially placed on a regular mesh in $\mathbb{R}^{2 d}$, then $(2.7)$ easily shows that just one particle will be located at a certain $x$-position, for almost every $t \in \mathbb{R}^{+}$. $\theta \tilde{w}$, evaluated at the position of this particle, $\left(x_{i}(t), v_{i}(t)\right)$, will therefore be zero for almost every $t$. Therefore, the kernel of the integral operator $\theta$, 
$\delta\left(x-x^{\prime}\right) \varphi\left(x^{\prime}, v-v^{\prime}\right)$, has to be smoothed in the $x$-direction. As in [12], we first replace $\theta$ by $\theta^{\varepsilon}$, which is also nonlocal in the $x$-direction:

$$
\left(\theta^{\varepsilon} w\right)(x, v)=\int_{\mathbb{R}_{x^{\prime}}^{d}} \int_{\mathbb{R}_{v^{\prime}}^{d}} \zeta^{\varepsilon}\left(x-x^{\prime}\right) \varphi\left(x^{\prime}, v-v^{\prime}\right) w\left(x^{\prime}, v^{\prime}\right) d x^{\prime} d v^{\prime} .
$$

There, the delta function is approximated by the cutoff function $\zeta^{\varepsilon}$, which is defined by

$$
\zeta^{\varepsilon}(x)=\frac{1}{\varepsilon^{d}} \zeta\left(\frac{x}{\varepsilon}\right)
$$

with $\zeta \in L^{1}\left(\mathbb{R}^{d}\right)$ and $\int_{\mathbb{R}^{d}} \zeta(x) d x=1$. Applying a quadrature approximation to $\theta^{\varepsilon}$, we now obtain the time evolution of the weights:

$$
\begin{gathered}
\frac{d w_{i}}{d t}=\sum_{j} \lambda_{j} \zeta^{\varepsilon}\left(x_{i}-x_{j}\right) \varphi\left(x_{j}, v_{i}-v_{j}\right) w_{j}, \\
w_{i}(t=0)=w_{I}\left(x_{i}(t=0), v_{i}(t=0)\right) .
\end{gathered}
$$

Note, that the right-hand side of $(2.9 \mathrm{a})$ is a discretization of the integral (2.8) with quadrature points at the positions of particles, $\left(x_{i}(t), v_{i}(t)\right), i \in \mathbb{Z}^{2 d}$, and $w\left(x_{i}(t), v_{i}(t), t\right)$ is approximated by $w_{i}(t)$.

This discretization is consistent when the integrand in (2.8) is regular enough. The proof requires certain regularity assumptions on $\zeta, \varphi$ and a priori estimates on the exact solution $w$ of the Wigner equation.

We remark that the Wigner equation is charge-conserving, i.e.,

$$
\int_{\mathbb{R}^{2 d}} w(t) d x d v=\int_{\mathbb{R}^{2 d}} w_{I} d x d v .
$$

This property carries over to the particle discretization, when the particles are initially distributed on a regular grid with equal phase space control volumes $\lambda_{i}=h^{2 d}, i \in \mathbb{Z}^{2 d}$, where $h$ denotes the mesh spacing. Since $\zeta^{\varepsilon}$ is even (see Hypothesis 1$)$ and $\varphi(x,-v)=-\varphi(x, v)$, the scheme can be made conservative:

$$
\begin{aligned}
\frac{d w_{i}}{d t}= & \sum_{j} \lambda_{j} \zeta^{\varepsilon}\left(x_{i}-x_{j}\right) \varphi\left(x_{j}, v_{i}-v_{j}\right) w_{j} \\
& -\sum_{j} \lambda_{j} \zeta^{\varepsilon}\left(x_{j}-x_{i}\right) \varphi\left(x_{i}, v_{j}-v_{i}\right) w_{i} \\
= & \sum_{j} \lambda_{j} \zeta^{\varepsilon}\left(x_{i}-x_{j}\right)\left[\varphi\left(x_{j}, v_{i}-v_{j}\right) w_{j}+\varphi\left(x_{i}, v_{j}-v_{i}\right) w_{i}\right] .
\end{aligned}
$$

Since the convergence analysis for this scheme is the same as for (2.9), and for reasons of simplicity, we shall only study the scheme (2.9).

The cutoff function can be chosen quite freely, and we shall assume

Hypothesis 1. The cutoff function $\zeta$ is an even compactly supported function such that

$$
\int_{\mathbb{R}_{x}^{d}} \zeta(x) d x=1 .
$$

Moreover, it satisfies for a fixed positive integer $r$ :

$$
\zeta \in W^{r, 1}\left(\mathbb{R}_{x}^{d}\right)
$$


and for all multi-indices $\alpha, 1 \leq|\alpha| \leq r-1$,

$$
\int_{\mathbb{R}_{x}^{d}} x^{\alpha} \zeta(x) d x=0
$$

Note that the function $\varphi$ is determined from the potential $V$. We will first impose rather strong assumptions on $V$ in order to develop a convergence analysis.

Hypothesis 2. The function $V$ belongs to $L^{2}\left(\mathbb{R}^{d}\right) \cap L^{\infty}\left(\mathbb{R}^{d}\right)$ and satisfies

$$
\int_{\mathbb{R}_{v}^{d}}\left|v^{\alpha}\right|\left|\partial^{\beta} \widehat{V}(v)\right| d v<+\infty \quad \forall(\alpha, \beta),|\alpha+\beta| \leq m,
$$

where $m$ is a fixed integer and $\widehat{V}$ denotes the Fourier transform of $V$. Moreover, there exist positive constants $C_{1}$ and $\varepsilon_{1}$ such that

$$
|\widehat{V}(v)| \leq \frac{C_{1}}{(1+|v|)^{d+\varepsilon_{1}}}
$$

These assumptions concern both the regularity and the decay of the function $V$ (expressed in terms of its Fourier transform $\widehat{V}$ ). The decay of the potential, which gives the regularity of $\widehat{V}$, is no crucial condition in our context, since $V$ shall describe a compactly supported potential barrier. But difficulties arise from the decay of the Fourier transform $\widehat{V}$, which corresponds to the regularity of $V$.

Especially for a discontinuous barrier of the form

$$
V(x)= \begin{cases}1 & \text { if } x \in\left[-\frac{1}{2}, \frac{1}{2}\right] \\ 0 & \text { else }\end{cases}
$$

estimates (2.11) and (2.12) no longer hold. The particle method for such a potential will be analyzed in $\S 5$ by mollifying the function $V$. But as a first step, we shall investigate the convergence of the particle method for a smooth potential $V$ which satisfies Hypothesis 2.

\section{A PRIORI ESTIMATES OF THE EXACT SOLUTION}

As in the previous section, we shall assume in the sequel that the potential $V$ belongs to $L^{2}\left(\mathbb{R}^{d}\right) \cap L^{\infty}\left(\mathbb{R}^{d}\right)$ and the initial data $w_{I}$ to $L^{2}\left(\mathbb{R}^{2 d}\right)$. This ensures existence and uniqueness of a mild solution in $L^{2}\left(\mathbb{R}^{2 d}\right)$ and entails the equivalence of equations (2.1) and (2.5). As we already mentioned, we need integrability and regularity of the exact solution $w$ in order to justify the quadrature approximation of the integral operator. All the estimates that we need will be expressed in terms of the usual norms of the spaces $L^{p}\left(\mathbb{R}^{2 d}\right)$ and $W^{m, p}\left(\mathbb{R}^{2 d}\right), 1 \leq p \leq \infty, m \in \mathbb{N}$, denoted by \|\|$_{L^{p}\left(\mathbb{R}^{2 d}\right)}$ and \|\|$_{W^{m, p}\left(\mathbf{R}^{2 d}\right)}$, respectively. We introduce the notation

$$
\||f|\|_{\mu, p}:=\sup _{|\alpha|+|\beta|+|\gamma|+|\delta| \leq \mu}\left\|x^{\alpha} v^{\beta} \partial_{x}^{\gamma} \partial_{v}^{\delta} f\right\|_{L^{p}\left(\mathbb{R}^{2 d}\right)}
$$

for $\mu \in \mathbb{N}_{0}$ and $1 \leq p \leq \infty$. 
Proposition 3.1. Let $\mu$ be an integer such that $0 \leq \mu \leq m$ ( $m$ given in Hypothesis 2) and $p$ a real number, $1 \leq p \leq \infty$.

Then, for any initial data $w_{I} \in L^{2}\left(\mathbb{R}^{2 d}\right)$ such that

$$
\left\|\mid w_{I}\right\|_{\mu, p}<\infty
$$

the mild $L^{2}\left(\mathbb{R}^{2 d}\right)$-solution $w$ of equation (2.1) satisfies

$$
\|w(t)\|\left\|_{\mu, p} \leq C(t)\right\|\left(1+|v|^{\mu}\right) \widehat{V}(v)\left\|_ { L ^ { 1 } ( \mathbb { R } ^ { d } ) } \left|\left\|w_{I} \mid\right\|_{\mu, p} .\right.\right.
$$

Here and in the proof of this proposition, $C(t)$ denotes (not necessarily equal) functions of $C([0, \infty))$ that may depend on $\mu$ and $p$.

The proof of this proposition is done in two steps. First, we introduce the strongly continuous group of operators $\left(G_{t}\right)_{t \in \mathbb{R}}$ generated by the drift term $-v \partial_{x}-E \partial_{v}$ of equation (2.5). It can be defined via a time-dependent change of variables,

$$
\left(G_{t} u\right)(x, v)=u(X(x, v, t), V(x, v, t)),
$$

related to the characteristics of the operator $-v \partial_{x}-E \partial_{v}$ :

$$
X(x, v, t)=x-v t+\frac{E t^{2}}{2}, \quad V(x, v, t)=v-E t .
$$

This group is a strongly continuous unitary group on any $L^{p}\left(\mathbb{R}^{2 d}\right), 1 \leq p<$ $\infty$ (see [17]). For $p=\infty$, however, $G_{t} u$ is not strongly continuous, but it still satisfies $\left\|G_{t} u\right\|_{L^{\infty}\left(\mathbb{R}^{2 d}\right)}=\|u\|_{L^{\infty}\left(\mathbb{R}^{2 d}\right)}$, which is sufficient for the following proofs. Moreover, $G_{t}$ satisfies:

Lemma 3.1. If a function $u$ satisfies \|\|$u \|_{\mu, p}<\infty$, then

$$
\|\| G_{t} u\left\|_{\mu, p} \leq C(t) \mid\right\| u \|_{\mu, p}
$$

holds for any $t \in \mathbb{R}$ and $1 \leq p \leq \infty$.

Secondly, the right-hand side of equation (2.5) is handled as a linear perturbation for which similar estimates hold.

Lemma 3.2. With the same assumptions as in Lemma 3.1, we have

$$
\left\|\varphi *_{v} u\right\|_{\mu, p} \leq C\left\|\left(1+|v|^{\mu}\right) \widehat{V}(v)\right\|_{L^{1}\left(\mathbb{R}^{d}\right)} \mid\|u\| \|_{\mu, p} .
$$

By setting $u(t)=G_{-t} w(t)$, equation (2.5) is transformed to

$$
\left\{\begin{array}{l}
\frac{d u}{d t}=G_{-t}\left(\varphi *_{v} G_{t} u\right), \\
u(0)=w_{I} .
\end{array}\right.
$$

Then, estimates (3.4) and (3.5) and Gronwall's lemma lead to

$$
\|u(t)\|_{\mu, p} \leq C(t)\left\|\left(1+|v|^{\mu}\right) \widehat{V}(v)\right\|_{L^{1}\left(\mathbb{R}^{d}\right)}||\left|w_{I}\right| \|_{\mu, p} .
$$

The proof of Proposition 3.1 ends by applying again estimate (3.4) with $w=G_{t} u$.

Proof of Lemma 3.1. Let $u$ be a function which satisfies estimate (3.1). The function defined by $f(t)=G_{t} u$ is the (distributional) solution of the transport equation

$$
\left\{\begin{array}{l}
\partial_{t} f+v \partial_{x} f+E \partial_{v} f=0 \\
f(t=0)=u
\end{array}\right.
$$


By applying the operator $x^{\alpha} v^{\beta} \partial_{x}^{\gamma} \partial_{v}^{\delta}$, we get

$$
\left\{\begin{array}{l}
\partial_{t}\left(x^{\alpha} v^{\beta} \partial_{x}^{\gamma} \partial_{v}^{\delta} f\right)+v \partial_{x}\left(x^{\alpha} v^{\beta} \partial_{x}^{\gamma} \partial_{v}^{\delta} f\right)+E \partial_{v}\left(x^{\alpha} v^{\beta} \partial_{x}^{\gamma} \partial_{v}^{\delta} f\right)=g(t), \\
\left(x^{\alpha} v^{\beta} \partial_{x}^{\gamma} \partial_{v}^{\delta} f\right)(t=0)=x^{\alpha} v^{\beta} \partial_{x}^{\gamma} \partial_{v}^{\delta} u
\end{array}\right.
$$

The right-hand side $g(t)$ is a sum of terms of the form $\left(x^{\alpha^{\prime}} v^{\beta^{\prime}} \partial_{x}^{\gamma^{\prime}} \partial_{v}^{\delta^{\prime}} f\right)(t)$, with $\left|\alpha^{\prime}\right|+\left|\beta^{\prime}\right|+\left|\gamma^{\prime}\right|+\left|\delta^{\prime}\right| \leq \mu$, and its $L^{p}$ norm is bounded by

$$
\|g(t)\|_{L^{p}\left(\mathbb{R}^{2 d}\right)} \leq C \mid\|f(t)\|_{\mu, p} .
$$

Thus, for any $t \in \mathbb{R}$, the function $\left(x^{\alpha} v^{\beta} \partial_{x}^{\gamma} \partial_{v}^{\delta} f\right)(t)$ is given by

$$
\left(x^{\alpha} v^{\beta} \partial_{x}^{\gamma} \partial_{v}^{\delta} f\right)(t)=G_{t}\left(x^{\alpha} v^{\beta} \partial_{x}^{\gamma} \partial_{v}^{\delta} u\right)+\int_{0}^{t} G_{t-s} g(s) d s .
$$

The inequalities

$$
\begin{aligned}
\left\|\left(x^{\alpha} v^{\beta} \partial_{x}^{\gamma} \partial_{v}^{\delta} f\right)(t)\right\|_{L^{p}\left(\mathbb{R}^{2 d}\right)} & \leq\left\|x^{\alpha} v^{\beta} \partial_{x}^{\gamma} \partial_{v}^{\delta} u\right\|_{L^{p\left(\mathbb{R}^{2 d}\right)}}+\int_{0}^{t}\|g(s)\|_{L^{p}\left(\mathbb{R}^{2 d}\right)} d s \\
& \leq\left\|x^{\alpha} v^{\beta} \partial_{x}^{\gamma} \partial_{v}^{\delta} u\right\|_{L^{p\left(\mathbb{R}^{2 d}\right)}}+C \int_{0}^{t}\|f(s)\| \|_{\mu, p} d s
\end{aligned}
$$

follow from the conservativity of the $L^{p}$ norm under the transformation $G_{t}$.

Then, Gronwall's lemma applied to

$$
\||f(t)|\|_{\mu, p} \leq\left|\|u\|_{\mu, p}+C \int_{0}^{t}\right|\|f(s) \mid\|_{\mu, p} d s
$$

ends the proof.

Proof of Lemma 3.2. Let $u$ be a function satisfying estimate (3.1). Let $\alpha, \beta, \gamma$, and $\delta$ be $d$-dimensional multi-indices such that $|\alpha|+|\beta|+|\gamma|+|\delta| \leq \mu$. We first compute the derivative

$$
\partial_{x}^{\gamma} \partial_{v}^{\delta}\left(\varphi *_{v} u\right)=\partial_{x}^{\gamma}\left(\varphi *_{v} \partial_{v}^{\delta} u\right) .
$$

Since the two functions depend on the $x$-variable, we use the Leibniz formula

$$
\partial_{x}^{\gamma} \partial_{v}^{\delta}\left(\varphi *_{v} u\right)=\sum_{\gamma_{1}+\gamma_{2}=\gamma} \frac{\gamma !}{\gamma_{1} ! \gamma_{2} !}\left[\left(\partial_{x}^{\gamma_{1}} \varphi\right) *_{v}\left(\partial_{x}^{\gamma_{2}} \partial_{v}^{\delta} u\right)\right] .
$$

When we multiply by $v^{\beta}$ we apply again Leibniz's formula:

$$
x^{\alpha} v^{\beta} \partial_{x}^{\gamma} \partial_{v}^{\delta}\left(\varphi *_{v} u\right)=\sum_{\substack{\beta_{1}+\beta_{2}=\beta \\ \gamma_{1}+\gamma_{2}=\gamma}} \frac{\beta ! \gamma !}{\beta_{1} ! \beta_{2} ! \gamma_{1} ! \gamma_{2} !}\left[\left(v^{\beta_{1}} \partial_{x}^{\gamma_{1}} \varphi\right) *_{v}\left(x^{\alpha} v^{\beta_{2}} \partial_{x}^{\gamma_{2}} \partial_{v}^{\delta} u\right)\right]
$$

Differentiating equality (2.4) with respect to $x$ gives

$$
\left(v^{\beta_{1}} \partial_{x}^{\gamma_{1}} \varphi\right)(x, v)=2\left(\frac{2}{\pi}\right)^{d / 2} v^{\beta_{1}} \operatorname{Im}\left[(2 i v)^{\gamma_{1}} e^{2 i v \cdot x} \widehat{V}(2 v)\right]
$$

and shows that $v^{\beta_{1}} \partial_{x}^{\gamma_{1}} \varphi$ belongs to $C\left(\mathbb{R}_{x}^{d}, L^{1}\left(\mathbb{R}_{v}^{d}\right)\right)$ with

$$
\left\|\left(v^{\beta_{1}} \partial_{x}^{\gamma_{1}} \varphi\right)(x, v)\right\|_{L^{1}\left(\mathbb{R}_{v}^{d}\right)} \leq C\left\|\left(1+|v|^{\mu}\right) \widehat{V}(v)\right\|_{L^{1}\left(\mathbb{R}^{d}\right)} \quad \forall x \in \mathbb{R}^{d} .
$$


Therefore, all the convolution products appearing in the sum (3.6) are defined and we have for almost every $x \in \mathbb{R}^{d}$

$$
\begin{aligned}
& \left\|\left(v^{\beta_{1}} \partial_{x}^{\gamma_{1}} \varphi\right) *_{v}\left(x^{\alpha} v^{\beta_{2}} \partial_{x}^{\gamma_{2}} \partial_{v}^{\delta} u\right)(x, v)\right\|_{L^{p}\left(\mathbb{R}_{v}^{d}\right)} \\
& \quad \leq C\left\|\left(1+|v|^{\mu}\right) \widehat{V}(v)\right\|_{L^{1}\left(\mathbb{R}^{d}\right)}\left\|\left(x^{\alpha} v^{\beta_{2}} \partial_{x}^{\gamma_{2}} \partial_{v}^{\delta} u\right)(x, v)\right\|_{L^{p}\left(\mathbb{R}_{v}^{d}\right)} .
\end{aligned}
$$

Taking the $L^{p}$ norm with respect to $x$ of both sides gives

$$
\begin{aligned}
& \left\|\left(v^{\beta_{1}} \partial_{x}^{\gamma_{1}} \varphi\right) *_{v}\left(x^{\alpha} v^{\beta_{2}} \partial_{x}^{\gamma_{2}} \partial_{v}^{\delta} u\right)\right\|_{L^{p}\left(\mathbb{R}^{2 d}\right)} \\
& \quad \leq C\left\|\left(1+|v|^{\mu}\right) \widehat{V}(v)\right\|_{L^{1}\left(\mathbb{R}^{d}\right)}\left\|x^{\alpha} v^{\beta_{2}} \partial_{x}^{\gamma_{2}} \partial_{v}^{\delta} u\right\|_{L^{p}\left(\mathbb{R}^{2 d}\right)} \\
& \quad \leq C\left\|\left(1+|v|^{\mu}\right) \widehat{V}(v)\right\|_{L^{1}\left(\mathbb{R}^{d}\right)}\|\| u\|\|_{\mu, p} .
\end{aligned}
$$

By inserting these estimates in (3.6), we get existence of a constant $C>0$ such that

$$
\left\|x^{\alpha} v^{\beta} \partial_{x}^{\gamma} \partial_{v}^{\delta}\left(\varphi *_{v} u\right)\right\|_{L^{p\left(\mathbb{R}^{2 d}\right)}} \leq C\left\|\left(1+|v|^{\mu}\right) \widehat{V}(v)\right\|_{L^{1}\left(\mathbb{R}^{d}\right)}\|u\| \|_{\mu, p}
$$

is valid for any $(\alpha, \beta, \gamma, \delta)$ with $|\alpha|+|\beta|+|\gamma|+|\delta| \leq \mu$.

Remark 3.1. We finish this section by noting that inequality (3.5) also holds for $\alpha=\beta=0$, and it then shows that the operator $w \rightarrow \varphi *_{v} w$ is a continuous linear operator in $W^{\mu, p}\left(\mathbb{R}^{2 d}\right)$. From this, standard semigroup theory [13] leads to existence and uniqueness of a solution of $(2.5)$ in $W^{\mu, p}\left(\mathbb{R}^{2 d}\right)$ when the initial data $w_{I}$ belongs to this space, without using the usual $L^{2}$-theory of the Wigner equation.

\section{CONVERgENCE OF THE PARTICLE METHOD FOR A SMOOTH POTENTIAL}

This section provides the convergence analysis for the method (2.7), (2.8). We assume here that Hypothesis 1 is satisfied with $r=m$ and that the potential $V$ has the regularity given by Hypothesis 2 with $m \geq 2 d$. The equations for the particle movement are easily solved:

$$
x_{i}(t)=x_{i}(0)+v_{i}(0) t+\frac{E t^{2}}{2}, \quad v_{i}(t)=v_{i}(0)+E t .
$$

Since these particles are initially distributed along a regular mesh (with mesh spacing $h$ ), they remain on a regular mesh deduced from the initial one by a linear mapping.

Given a particle approximation (2.6) of the solution $w$, with $w_{i}(t) \simeq$ $w\left(x_{i}(t), v_{i}(t), t\right)$, one can easily construct a smooth approximation of $w$ :

$$
w_{\varepsilon}^{h}(x, v, t)=\sum_{i \in \mathbb{Z}^{2 d}} h^{2 d} w_{i}(t) \zeta^{\varepsilon}\left(x-x_{i}(t)\right) \zeta^{\varepsilon}\left(v-v_{i}(t)\right),
$$

where the delta functions of (2.6) have been regularized by the cutoff $\zeta^{\varepsilon}$.

The crucial point of the convergence analysis is the error estimate of the approximation $w\left(x_{i}(t), v_{i}(t), t\right) \simeq w_{i}(t)$, which is given by the following theorem.

Theorem 4.1. Let the initial data $w_{I}$ belong to $L^{2}\left(\mathbb{R}^{2 d}\right) \cap W^{m, \infty}\left(\mathbb{R}^{2 d}\right)$ and satisfy $\left\|\left|w_{I}\right|\right\|_{m, \infty}<\infty$. Then, for any $T>0$, there exists a positive constant $C(T, m, V, \zeta)$ so that

$$
\begin{aligned}
& \sup _{i \in \mathbb{Z}^{2 d}}\left|w\left(x_{i}(t), v_{i}(t), t\right)-w_{i}(t)\right| \\
& \quad \leq C(T, m, V, \zeta)||\left|w_{I}\right| \|_{m, \infty}\left(\varepsilon^{m}+\frac{h^{m}}{\varepsilon^{m}}\right)
\end{aligned}
$$

holds for any $t, t \in[0, T]$. 
Here, $\varepsilon$ denotes the scaling parameter of the cutoff function $\zeta$ and $h$ the grid spacing of the regular particle mesh. From this result, standard particle method analysis [10] leads to the error estimate

$$
\sup _{t \in[0, T]}\left\|w(t)-w_{\varepsilon}^{h}(t)\right\|_{L^{\infty}\left(\mathbb{R}^{2 d}\right)}=O\left(\varepsilon^{m}+\frac{h^{m}}{\varepsilon^{m}}\right) .
$$

As usual for particle methods, the theorem will be proved in two steps:

- consistency of the quadrature approximation involved in (2.9a),

- stability of the differential system (2.9).

4.1. Consistency. The quadrature formula (2.9a) relies on two approximations: the regularization of the operator $\theta$ and the approximation of the integral (2.8) by a discrete sum. First, the regularization consists in replacing $\theta w$ by

$$
\theta^{\varepsilon} w=\zeta^{\varepsilon} *_{x}\left(\varphi *_{v} w\right)
$$

Since the cutoff function $\zeta^{\varepsilon}$ satisfies Hypothesis 1, the following result [10] holds.

Lemma 4.1. If $u \in W^{m, p}\left(\mathbb{R}^{d}\right), 1 \leq p \leq \infty$, then we have

$$
\left\|u-\zeta^{\varepsilon} * u\right\|_{L^{p}\left(\mathbb{R}^{d}\right)} \leq C \varepsilon^{m}\|u\|_{W^{m, p}\left(\mathbb{R}^{d}\right)} .
$$

Proposition 3.1 and Lemma 3.1 show that $w$ and $\theta w$ belong to the space $W^{m, \infty}\left(\mathbb{R}^{2 d}\right)$, which is included in $W^{m, \infty}\left(\mathbb{R}_{x}^{d}, L^{\infty}\left(\mathbb{R}_{v}^{d}\right)\right)$. Therefore, the previous lemma implies the estimate

$$
\left\|\theta w-\theta^{\varepsilon} w\right\|_{L^{\infty}\left(\mathbb{R}_{x}^{d}, L^{\infty}\left(\mathbb{R}_{v}^{d}\right)\right)} \leq C \varepsilon^{m}\|\theta w\|_{W^{m, \infty}\left(\mathbb{R}_{x}^{d}, L^{\infty}\left(\mathbb{R}_{v}^{d}\right)\right)},
$$

and hence, by Remark 3.1, the following lemma.

Lemma 4.2. Under the assumptions of Theorem 4.1 we have

$$
\left\|\theta w-\theta^{\varepsilon} w\right\|_{L^{\infty}\left(\mathbb{R}^{2 d}\right)} \leq C(t) \varepsilon^{m}\left\|w_{I}\right\|_{W^{m, \infty}\left(\mathbb{R}^{2 d}\right)},
$$

with $C \in C([0, \infty))$.

Next, we investigate the error related to the discretization of the integral by applying a standard result $[10,14]$ :

Lemma 4.3. Let $\left(x_{i}, v_{i}\right), i \in \mathbb{Z}^{2 d}$, be a mesh in $\mathbb{R}^{2 d}$ with control volumes $\omega_{i}$, $i \in \mathbb{Z}^{2 d}$, and mesh spacing $h\left(\omega_{i}=O\left(h^{2 d}\right)\right)$. Then, there exists a constant $C>0$, independent of $h$, such that for any function $g \in W^{m, 1}\left(\mathbb{R}^{2 d}\right), m \geq 2 d$, we have

$$
\left|\iint g(x, v) d x d v-\sum_{i} \omega_{i} g\left(x_{i}, v_{i}\right)\right| \leq C h^{m}\|g\|_{W^{m, 1}\left(\mathbb{R}^{2 d}\right)} .
$$

So it only remains to prove that the integrand in (2.8),

$$
g(x, v)=\zeta^{\varepsilon}\left(x_{0}-x\right) \varphi\left(x, v_{0}-v\right) w\left(x, v, t_{0}\right), \quad\left(x_{0}, v_{0}, t_{0}\right) \text { fixed },
$$

belongs to $W^{m, 1}\left(\mathbb{R}^{2 d}\right)$. 
Lemma 4.4. For any $T>0$, there exists a constant $C(T, m, V, \zeta)$ so that

$$
\begin{gathered}
\left\|\zeta^{\varepsilon}\left(x_{0}-x\right) \varphi\left(x, v_{0}-v\right) w\left(x, v, t_{0}\right)\right\|_{W^{m, 1}\left(\mathbb{R}^{2 d}\right)} \\
\leq C(T, m, V, \zeta)\left\|w_{I}\right\|_{m, \infty} \frac{1}{\varepsilon^{m}}
\end{gathered}
$$

holds for any $\varepsilon>0$ and for any $\left(x_{0}, v_{0}, t_{0}\right) \in \mathbb{R}^{2 d} \times[0, T]$.

Proof. Let $\left(x_{0}, v_{0}, t_{0}\right)$ belong to $\mathbb{R}^{2 d} \times[0, T]$ and $\varepsilon$ be a positive real number. We consider the function $g$ defined by (4.4) and we compute its derivatives $\partial_{x}^{\alpha} \partial_{v}^{\beta} g,|\alpha|+|\beta| \leq m$. First, we differentiate with respect to $v$ and use equality (2.4) for $\varphi$ :

$$
\begin{aligned}
\partial_{v}^{\beta} g(x, v)= & \sum_{\beta_{1}+\beta_{2}=\beta} \frac{\beta !}{\beta_{1} ! \beta_{2} !} \zeta^{\varepsilon}\left(x_{0}-x\right)(-1)^{\beta_{1}} \partial_{v}^{\beta_{1}} \varphi\left(x, v_{0}-v\right) \partial_{v}^{\beta_{2}} w\left(x, v, t_{0}\right) \\
= & \sum_{\beta_{1}+\beta_{2}+\beta_{3}=\beta} \frac{\beta !}{\beta_{1} ! \beta_{2} ! \beta_{3} !} \zeta^{\varepsilon}\left(x_{0}-x\right) 2\left(\frac{2}{\pi}\right)^{d / 2} \\
& \times \operatorname{Im}\left[(-2 i x)^{\beta_{1}} e^{2 i x \cdot\left(v_{0}-v\right)}(-2)^{\beta_{2}} \partial_{v}^{\beta_{2}} \widehat{V}\left(2\left(v_{0}-v\right)\right)\right] \partial_{v}^{\beta_{3}} w\left(x, v, t_{0}\right) .
\end{aligned}
$$

Secondly, differentiating with respect to $x$ gives

$$
\begin{aligned}
& \partial_{x}^{\alpha} \partial_{v}^{\beta} g(x, v) \\
& =\sum_{\substack{\alpha_{1}+\alpha_{2}+\alpha_{3}+\alpha_{4}=\alpha \\
\beta_{1}+\beta_{2}+\beta_{3}=\beta \\
\alpha_{2} \leq \beta_{1}}}\left\{\frac{\alpha ! \beta !}{\alpha_{1} ! \alpha_{2} ! \alpha_{3} ! \alpha_{4} ! \beta_{1} ! \beta_{2} ! \beta_{3} !}(-1)^{\alpha_{1}} \partial_{x}^{\alpha_{1}} \zeta^{\varepsilon}\left(x_{0}-x\right)\right. \\
& \quad \times 2\left(\frac{2}{\pi}\right)^{d / 2} \operatorname{Im}\left[(-2 i)^{\beta_{1}} \frac{\beta_{1} !}{\left(\beta_{1}-\alpha_{2}\right) !} x^{\left(\beta_{1}-\alpha_{2}\right)}\left[2 i\left(v_{0}-v\right)\right]^{\alpha_{3}} e^{2 i x \cdot\left(v_{0}-v\right)}\right. \\
& \left.\left.\times(-2)^{\beta_{2}} \partial_{v}^{\beta_{2}} \widehat{V}\left(2\left(v_{0}-v\right)\right)\right] \partial_{x}^{\alpha_{4}} \partial_{v}^{\beta_{3}} w\left(x, v, t_{0}\right)\right\} .
\end{aligned}
$$

The $L^{1}$ norm of this derivative is estimated by

$$
\begin{aligned}
\left\|\partial_{x}^{\alpha} \partial_{v}^{\beta} g\right\|_{L^{1}\left(\mathbb{R}^{2 d}\right)} \leq C(\alpha, \beta) \sum_{\substack{\alpha_{1}+\alpha_{2}+\alpha_{3}+\alpha_{4}=\alpha \\
\beta_{1}+\beta_{2}+\beta_{3}=\beta \\
\alpha_{2} \leq \beta_{1}}}\left[\left\|\partial_{x}^{\alpha_{1}} \zeta^{\varepsilon}(x)\right\|_{L^{1}\left(\mathbb{R}^{d}\right)}\left\|v^{\alpha_{3}} \partial_{v}^{\beta_{2}} \widehat{V}(v)\right\|_{L^{1}\left(\mathbb{R}^{d}\right)}\right. \\
\left.\quad \times\left\|x^{\beta_{1}-\alpha_{2}} \partial_{x}^{\alpha_{4}} \partial_{v}^{\beta_{3}} w\left(x, v, t_{0}\right)\right\|_{L^{\infty}\left(\mathbb{R}^{2 d}\right)}\right] .
\end{aligned}
$$

Then Proposition 3.1 leads to

$$
\begin{aligned}
\|g\|_{W^{m, 1}\left(\mathbb{R}^{2 d}\right)} \leq & C(m, t)\left\|\zeta^{\varepsilon}(x)\right\|_{W^{m, 1}\left(\mathbb{R}^{d}\right)} \\
& \times\left[\sup _{|\alpha|+|\beta| \leq m}\left\|v^{\alpha} \partial_{v}^{\beta} \widehat{V}(v)\right\|_{L^{1}\left(\mathbb{R}^{2 d}\right)}\right]^{2}\left\|w_{I}\right\| \|_{m, \infty},
\end{aligned}
$$

with $C(m, \cdot) \in C([0, \infty))$.

Finally, estimate (4.5) is obtained with

$$
\left\|\zeta^{\varepsilon}\right\|_{W^{m, 1}\left(\mathbb{R}^{d}\right)} \leq \frac{C}{\varepsilon^{m}}\|\zeta\|_{W^{m, 1}\left(\mathbb{R}^{d}\right)} .
$$

The following proposition is an immediate consequence of the previous lemmas and concludes the consistency analysis. 
Proposition 4.1. Under the assumptions of Theorem 4.1 and for any $T>0$, there exists a constant $C(T, m, V, \zeta)$ so that

$$
\begin{aligned}
& \left|\theta w\left(x_{0}, v_{0}, t_{0}\right)-\sum_{j \in \mathbb{Z}^{2 d}} h^{2 d} \zeta^{\varepsilon}\left(x_{0}-x_{j}\right) \varphi\left(x_{j}, v_{0}-v_{j}\right) w\left(x_{j}, v_{j}, t_{0}\right)\right| \\
& \quad \leq C(T, m, V, \zeta)||\left|w_{I}\right|||_{m, \infty}\left(\varepsilon^{m}+\frac{h^{m}}{\varepsilon^{m}}\right)
\end{aligned}
$$

holds for any $\left(x_{0}, v_{0}, t_{0}\right) \in \mathbb{R}^{2} \times[0, T]$.

Proof. Writing

$$
\begin{aligned}
& \left|\theta w\left(x_{0}, v_{0}, t_{0}\right)-\sum_{j \in \mathbb{Z}^{2 d}} h^{2 d} \zeta^{\varepsilon}\left(x_{0}-x_{j}\right) \varphi\left(x_{j}, v_{0}-v_{j}\right) w\left(x_{j}, v_{j}, t_{0}\right)\right| \\
& \leq\left|\theta w\left(x_{0}, v_{0}, t_{0}\right)-\theta^{\varepsilon} w\left(x_{0}, v_{0}, t_{0}\right)\right| \\
& \quad+\left|\theta^{\varepsilon} w\left(x_{0}, v_{0}, t_{0}\right)-\sum_{j \in \mathbb{Z}^{2 d}} h^{2 d} \zeta^{\varepsilon}\left(x_{0}-x_{j}\right) \varphi\left(x_{j}, v_{0}-v_{j}\right) w\left(x_{j}, v_{j}, t_{0}\right)\right|
\end{aligned}
$$

and applying Lemma 4.2 to the first term, and Lemmas 4.3 and 4.4 to the second term, yields the result.

4.2. Stability. The weighted particle method reduces to a system of infinitely many differential equations

$$
\frac{d w_{i}}{d t}=\sum_{j \in \mathbb{Z}^{2 d}} h^{2 d} \zeta^{\varepsilon}\left(x_{i}-x_{j}\right) \varphi\left(x_{j}, v_{i}-v_{j}\right) w_{j}, \quad i \in \mathbb{Z}^{2 d},
$$

while the exact solution satisfies

$$
\frac{d}{d t}\left[w\left(x_{i}(t), v_{i}(t), t\right)\right]=(\theta w)\left(x_{i}(t), v_{i}(t), t\right), \quad i \in \mathbb{Z}^{2 d}
$$

We set

$$
e_{i}(t)=w\left(x_{i}(t), v_{i}(t), t\right)-w_{i}(t), \quad i \in \mathbb{Z}^{2 d} .
$$

The $e_{i}$ satisfy

$$
\begin{aligned}
& \frac{d}{d t} e_{i}=\sum_{j \in \mathbb{Z}^{2 d}} h^{2 d} \zeta^{\varepsilon}\left(x_{i}-x_{j}\right) \varphi\left(x_{j}, v_{i}-v_{j}\right) e_{j} \\
& +\left[(\theta w)\left(x_{i}, v_{i}, t\right)\right. \\
& \left.-\sum_{j \in \mathbb{Z}^{2 d}} h^{2 d} \zeta^{\varepsilon}\left(x_{i}-x_{j}\right) \varphi\left(x_{j}, v_{i}-v_{j}\right) w\left(x_{j}, v_{j}, t\right)\right] \text {. }
\end{aligned}
$$

We aim at establishing a uniform (in $i$ and $t$ ) estimate of the error $e_{i}$, as stated in Theorem 4.1. We already know from the consistency result in Proposition 4.1 that the last term in (4.9) is of order $O\left(\varepsilon^{m}+h^{m} / \varepsilon^{m}\right)$. The last ingredient is then the stability of the differential system (4.9) or (4.7), which is based on the next lemma. 
Lemma 4.5. Let $t$ belong to $[0, T]$ and $L=L(t)$ be an operator on $l^{p}\left(\mathbb{Z}^{2 d}\right)$, $1 \leq p \leq \infty$, defined by

$$
(L(t) u)_{i}=\sum_{j \in \mathbb{Z}^{2 d}} h^{2 d} \zeta^{\varepsilon}\left(x_{i}(t)-x_{j}(t)\right) \varphi\left(x_{j}(t), v_{i}(t)-v_{j}(t)\right) u_{j}, \quad i \in \mathbb{Z}^{2 d},
$$

for any $u=\left(u_{i}\right)_{i \in \mathbb{Z}^{2 d}}, u \in l^{p}\left(\mathbb{Z}^{2 d}\right)$.

Under Hypotheses 1 and $2, L$ is a continuous linear operator in $l^{p}\left(\mathbb{Z}^{2 d}\right)$, uniformly bounded with respect to $t \in[0, T], \varepsilon<1, h<1,1 \leq p \leq \infty$.

Proof. We will directly prove the lemma for $p=1$ and $p=\infty$. Then, the Riesz-Thorin interpolation theorem [20] yields the general result.

First let $u$ belong to $l^{\infty}\left(\mathbb{Z}^{2 d}\right)$. For any $i \in \mathbb{Z}^{2 d}$, we have

$$
\begin{aligned}
\left|(L u)_{i}\right| & \leq \sum_{j \in \mathbb{Z}^{2 d}} h^{2 d}\left|\zeta^{\varepsilon}\left(x_{i}-x_{j}\right)\right|\left|\varphi\left(x_{j}, v_{i}-v_{j}\right)\right|\left|u_{j}\right| \\
& \leq 2\left(\frac{2}{\pi}\right)^{d / 2} \sum_{j \in \mathbb{Z}^{2 d}} \frac{h^{2 d}}{\varepsilon^{d}}\left|\zeta\left(\frac{x_{i}-x_{j}}{\varepsilon}\right)\right|\left|\widehat{V}\left(2\left(v_{i}-v_{j}\right)\right)\right|\left|u_{j}\right| \\
& \leq M \frac{h^{2 d}}{\varepsilon^{d}}\left[\sum_{j \in \mathbb{Z}^{2 d}: \zeta^{\varepsilon}\left(x_{i}-x_{j}\right) \neq 0}\left|\widehat{V}\left(2\left(v_{i}-v_{j}\right)\right)\right|\right]\|u\|_{l^{\infty}\left(\mathbb{Z}^{2 d}\right)},
\end{aligned}
$$

with $M=2(2 / \pi)^{d / 2} \sup _{x \in \mathbb{R}^{d}}|\zeta(x)|$.

Equalities (4.0) easily show that the distance between two particles remains uniformly bounded from below:

$$
\forall t \in[0, T], \quad h \leq \min _{\substack{j \neq i \\ i, j \in \mathbb{Z}^{2 d}}}\left\{\left|x_{i}(t)-x_{j}(t)\right|+\left|v_{i}(t)-v_{j}(t)\right|\right\} .
$$

Then, for any $i \in \mathbb{Z}^{2 d}$ and any $k \in \mathbb{N}$, the number $N_{j}^{k}(t)$ of particles in the domain

$$
\Omega_{i}^{k}(t)=\left\{x \in \mathbb{R}^{d} \mid \zeta^{\varepsilon}\left(x_{i}-x\right) \neq 0\right\} \times\left\{v \in \mathbb{R}^{d}|k-1 \leq| v_{i}-v \mid \leq k\right\}
$$

is bounded by

$$
N_{i}^{k}(t) \leq C_{2} \frac{\varepsilon^{d}}{h^{2 d}} k^{d-1},
$$

where $C_{2}$ is a constant independent of $i \in \mathbb{Z}^{2 d}, k \in \mathbb{N}$, and $t \in[0, T]$. Since $\widehat{V}$ satisfies

$$
|\widehat{V}(v)| \leq \frac{C_{1}}{(1+|v|)^{d+\varepsilon_{1}}}, \quad \varepsilon_{1}>0
$$

we thus have for any $i \in \mathbb{Z}^{2 d}$ :

$$
\begin{aligned}
\sum_{j \in \mathbb{Z}^{2 d}: \zeta^{\varepsilon}\left(x_{i}-x_{j}\right) \neq 0}\left|\widehat{V}\left(2\left(v_{i}-v_{j}\right)\right)\right| & \leq C_{1} C_{2} \frac{\varepsilon^{d}}{h^{2 d}}\left(\sum_{k=1}^{\infty} \frac{k^{d-1}}{(2 k-1)^{d+\varepsilon_{1}}}\right) \\
& \leq C_{3} \frac{\varepsilon^{d}}{h^{2 d}} .
\end{aligned}
$$

We insert this estimate in (4.10) and obtain

$$
\left|(L u)_{i}\right| \leq M C_{3}\|u\|_{l^{\infty}\left(\mathbb{Z}^{2 d}\right)} \quad \forall i \in \mathbb{Z}^{2 d}, t \in[0, T] .
$$


For $u \in l^{1}\left(\mathbb{Z}^{2 d}\right)$ we analogously obtain

$$
\begin{aligned}
\|L u\|_{l^{\prime}\left(\mathbb{Z}^{2 d}\right)} & =\sum_{i \in \mathbb{Z}^{2 d}}\left|(L u)_{i}\right| \\
& \leq M \frac{h^{2 d}}{\varepsilon^{d}} \sum_{i \in \mathbb{Z}^{2 d}}\left[\sum_{j \in \mathbb{Z}^{2 d}: \zeta^{\varepsilon}\left(x_{i}-x_{j}\right) \neq 0}\left|\widehat{V}\left(2\left(v_{i}-v_{j}\right)\right)\right|\left|u_{j}\right|\right] .
\end{aligned}
$$

Since $|\widehat{V}(-v)|=|\widehat{V}(v)|$, we can reorder this sum and apply (4.11):

$$
\begin{aligned}
\|L u\|_{l^{1}\left(\mathbb{Z}^{2 d}\right)} & \leq M \frac{h^{2 d}}{\varepsilon^{d}} \sum_{i \in \mathbb{Z}^{2 d}}\left|u_{i}\right|\left[\sum_{j \in \mathbb{Z}^{2 d}}: \zeta_{\zeta^{\varepsilon}\left(x_{i}-x_{j}\right) \neq 0}\left|\widehat{V}\left(2\left(v_{i}-v_{j}\right)\right)\right|\right] \\
& \leq M C_{3}\|u\|_{l^{1}\left(\mathbb{Z}^{2 d}\right)}, \quad t \in[0, T] .
\end{aligned}
$$

This lemma yields

Proposition 4.2. The differential equations (4.7) and (4.9) admit unique solutions in $l^{\infty}\left(\mathbb{Z}^{2 d}\right)$.

Moreover, the proof of Theorem 4.1 ends by applying Gronwall's lemma to the differential system (4.9).

4.3. $L^{p}$-convergence. First we want to derive the $l^{p}$-convergence of the particle approximation $w_{i}$. Since each particle has the phase space control volume $h^{2 d}$, we have to consider the following $h$-dependent norms:

$$
\|e\|_{l_{h}^{p}\left(\mathbb{Z}^{2 d}\right)}:=\left(\sum_{i \in \mathbb{Z}^{2 d}} h^{2 d}\left|e_{i}\right|^{p}\right)^{1 / p}, \quad 1 \leq p<\infty .
$$

Theorem 4.2. Let the initial data $w_{I}$ belong to $L^{2}\left(\mathbb{R}^{2 d}\right) \cap W^{m, \infty}\left(\mathbb{R}^{2 d}\right)$ and satisfy (3.1) with $\mu=m$ and $p=\infty$. Then, for any $T>0$ and any $\varepsilon_{2}>0$, there exists a positive constant $C_{p, \varepsilon_{2}}$ such that

$$
\|e(t)\|_{l_{h}^{p}\left(\mathbb{Z}^{2 d}\right)} \leq C_{p, \varepsilon_{2}}\left(\varepsilon^{m}+\frac{h^{m}}{\varepsilon^{m}}\right)^{1-\left(1+\varepsilon_{2}\right) / p}, \quad 1<p<\infty,
$$

holds for any $t \in[0, T]$.

Proof. The error of the particle method, defined by

$$
e_{i}(t)=w\left(x_{i}(t), v_{i}(t), t\right)-w_{i}(t), \quad i \in \mathbb{Z}^{2 d},
$$

already satisfies $\|e\|_{l \infty}\left(\mathbb{Z}^{2 d}\right) \rightarrow 0$ when $h / \varepsilon, \varepsilon \rightarrow 0$. Therefore, it is sufficient to prove uniform boundedness of $\|e\|_{h}^{p}\left(\mathbb{Z}^{2 d}\right), 1<p<\infty$, and to apply the following estimate:

$$
\|e\|_{l_{h}^{p}\left(\mathbb{Z}^{2 d}\right)}^{p} \leq\|e\|_{l^{\infty}\left(\mathbb{Z}^{2 d}\right)}^{p-1-\varepsilon^{d}}\|e\|_{l_{h}^{1+\varepsilon_{2}}\left(\mathbb{Z}^{2 d}\right)}^{1+\varepsilon_{2}} .
$$

From Proposition 3.1 we readily conclude

$$
\left|w\left(x_{i}(t), v_{i}(t), t\right)\right| \leq \frac{C}{1+\left|x_{i}(t)\right|^{2 d}+\left|v_{i}(t)\right|^{2 d}},
$$


with $C$ independent of $i \in \mathbb{Z}^{2 d}$ and $t \in[0, T]$. Since the particles are always positioned on a regular grid, uniformly covering the phase space $\mathbb{R}^{2 d}$ (see (4.0)), (4.17) represents a decay estimate, which implies

$$
\left.\left\|w\left(x_{i}(t), v_{i}(t), t\right)\right\|_{h}^{p} \mathbb{Z}^{2 d}\right) \leq C_{p}, \quad 1<p<\infty,
$$

with $C_{p}$ independent of $h<1, t \in[0, T]$.

Since Lemma 4.5 implies the $l_{h}^{p}$-stability of the differential equation (4.7), we conclude that $\left(w_{i}\right)_{i \in \mathbb{Z}^{2 d}}$, and thus $e$, are bounded in $l_{h}^{p}\left(\mathbb{Z}^{2 d}\right), 1<p<\infty$, uniformly with respect to $t \in[0, T], \varepsilon<1, h<1$.

We remark that a convergence result for the $l_{h}^{1}\left(\mathbb{Z}^{2 d}\right)$-norm can be obtained if stricter conditions on $w_{I}$ are imposed.

From this theorem we can now obtain $L^{p}\left(\mathbb{R}^{2 d}\right)$-convergence of $w_{\varepsilon}^{h}$, defined in (4.1a):

Theorem 4.3. Under the assumptions of Theorem 4.2 and for any $\varepsilon_{2}>0$, there exists a positive constant $C_{p, \varepsilon_{2}}$ such that

$$
\begin{aligned}
\sup _{t \in[0, T]}\left\|w(t)-w_{\varepsilon}^{h}(t)\right\|_{L^{p}\left(\mathbb{R}^{2 d}\right)} & \\
\leq & C_{p, \varepsilon_{2}}\left(\varepsilon^{m}+\frac{h^{m}}{\varepsilon^{m}}\right)^{1-\left(1+\varepsilon_{2}\right) / p}, \quad 1<p \leq \infty .
\end{aligned}
$$

Proof. The result for $p=\infty$ has already been obtained in $\S 4$.2. For $1<p<\infty$, we estimate

$$
\begin{aligned}
& \left\|w(t)-w_{\varepsilon}^{h}(t)\right\|_{L^{p}\left(\mathbb{R}^{2 d}\right)} \\
& \quad \leq\left\|w(t)-\Pi_{\varepsilon}^{h}(t) w(t)\right\|_{L^{p}\left(\mathbb{R}^{2 d}\right)}+\left\|\Pi_{\varepsilon}^{h}(t) w(t)-w_{\varepsilon}^{h}(t)\right\|_{L^{p}\left(\mathbb{R}^{2 d}\right)},
\end{aligned}
$$

where the smoothed particle approximation of $w(t)$ is defined by

$$
\Pi_{\varepsilon}^{h}(t) w(t):=\sum_{i \in \mathbb{Z}^{2 d}} h^{2 d} w\left(x_{i}(t), v_{i}(t), t\right) \zeta^{\varepsilon}\left(x-x_{i}(t)\right) \zeta^{\varepsilon}\left(v-v_{i}(t)\right) .
$$

Standard particle method analysis [10] shows that the first term of the righthand side of (4.20) is $O\left(\varepsilon^{m}+h^{m} / \varepsilon^{m}\right)$. Since $\zeta$ is compactly supported and $\left|\zeta^{\varepsilon}(x)\right| \leq M / \varepsilon^{d}$, we estimate the second term as follows:

$$
\begin{gathered}
\int_{\mathbb{R}^{2 d}}\left|\sum_{i \in \mathbb{Z}^{2 d}} h^{2 d}\left[w\left(x_{i}, v_{i}, t\right)-w_{i}(t)\right] \zeta^{\varepsilon}\left(x-x_{i}\right) \zeta^{\varepsilon}\left(v-v_{i}\right)\right|^{p} d x d v \\
\quad \leq \int_{\mathbb{R}^{2 d}}\left[M \frac{h^{2 d}}{\varepsilon^{2 d}} \sum_{\substack{\left|x_{i}-x\right| \leq C \varepsilon \\
\left|v_{i}-v\right| \leq C \varepsilon}}\left|w\left(x_{i}, v_{i}, t\right)-w_{i}(t)\right|\right]^{p} d x d v \\
\quad \leq \sum_{j \in \mathbb{Z}^{2 d}} h^{2 d}\left[M \frac{h^{2 d}}{\varepsilon^{2 d}} \sum_{\substack{\left|x_{i}-x_{j}\right| \leq C(\varepsilon+h) \\
\left|v_{i}-v_{j}\right| \leq C(\varepsilon+h)}}\left|w\left(x_{i}, v_{i}, t\right)-w_{i}(t)\right|\right]^{p} .
\end{gathered}
$$

In this last expression the sum over $i$ is finite, including at most $N=$ $C\left(1+\varepsilon^{2 d} / h^{2 d}\right)$ particles, with $C$ independent of $j, \varepsilon, h$, and $t$. Using the 
estimate $\left(\sum_{i=1}^{N}\left|a_{i}\right|\right)^{p} \leq N^{p-1} \sum_{i=1}^{N}\left|a_{i}\right|^{p}$, we obtain

$$
\begin{aligned}
& \left\|\Pi_{\varepsilon}^{h}(t) w(t)-w_{\varepsilon}^{h}(t)\right\|_{L^{p}\left(\mathbb{R}^{2 d}\right)}^{p} \\
& \leq C M^{p} \sum_{j \in \mathbb{Z}^{2 d}}\left(\frac{h}{\varepsilon}\right)^{2 d p} N^{p-1} \sum_{\substack{\left|x_{i}-x_{j}\right| \leq C(\varepsilon+h) \\
\left|v_{i}-v_{j}\right| \leq C(\varepsilon+h)}} h^{2 d}\left|w\left(x_{i}, v_{i}, t\right)-w_{i}(t)\right|^{p} .
\end{aligned}
$$

Since each term $\left|w\left(x_{i}, v_{i}, t\right)-w_{i}(t)\right|^{p}, i \in \mathbb{Z}^{2 d}$, can only appear $N$ times in this expression, we finally get

$$
\left\|\Pi_{\varepsilon}^{h}(t) w(t)-w_{\varepsilon}^{h}(t)\right\|_{L^{p}\left(\mathbb{R}^{2 d}\right)}^{p} \leq C M^{p}\left(1+\frac{h^{2 d}}{\varepsilon^{2 d}}\right)^{p}\|e\|_{l_{h}^{p}\left(\mathbb{Z}^{2 d}\right)}^{p} .
$$

\section{CONVERGENCE ANALYSIS FOR A RECTANGULAR POTENTIAL BARRIER}

In this section, we shall analyze the one-dimensional particle method for the potential $V$, given by

$$
V(x)= \begin{cases}1 & \text { if } x \in\left(-\frac{1}{2}, \frac{1}{2}\right) \\ 0 & \text { else }\end{cases}
$$

Its Fourier transform is

$$
\widehat{V}(v)=\frac{1}{\sqrt{2 \pi}} \frac{\sin (v / 2)}{v / 2} .
$$

Then, Hypothesis 2 and, consequently, the above convergence analysis, no longer hold. In order to solve this problem, we propose two different methods. The first consists in slightly modifying the original equation by taking a mollified potential $V^{\alpha}=V * \eta^{\alpha}$. Then we prove that the exact solution $w^{\alpha}$ of the Wigner equation with the potential $V^{\alpha}$ converges in $L^{2}\left(\mathbb{R}^{2}\right)$ to the solution $w$ obtained with $V$, when $\alpha \rightarrow 0$. The particle method (2.7), (2.9) is applied to the regularized equation, for which Theorem 4.3 ensures convergence in $L^{2}\left(\mathbb{R}^{2}\right)$. Then the combination of these two approximations yields convergence in $L^{2}\left(\mathbb{R}^{2}\right)$ as $\alpha \rightarrow 0, \varepsilon \rightarrow 0, h / \varepsilon \rightarrow 0$. The second way to treat the rectangular potential relies on a modified particle method which preserves the discrete $l^{2}$-norm of the particle solution. It will be directly applied to the Wigner equation for the nonsmooth potential $V$.

In both cases, we will assume that the initial data $w_{I}$ belongs to $L^{2}\left(\mathbb{R}^{2}\right)$, which implies existence and uniqueness of a solution $w(t) \in C\left(\mathbb{R}, L^{2}\left(\mathbb{R}^{2}\right)\right)$. The time-variable will be confined to a fixed interval $[0, T]$ and we notice that the set $\{w(t), t \in[0, T]\}$ is a compact subset of $L^{2}\left(\mathbb{R}^{2}\right)$, as the continuous image of $[0, T]$. This compactness leads to $L^{2}$-strong convergence results for the two methods that we propose. In particular, we will refer to the following lemma:

Lemma 5.1. Let $E$ denote a Banach space. If the sequence $\left(S_{n}\right)_{n \in \mathbb{N}}$ of bounded linear operators converges to $S$ in the strong operator topology, then it converges to $S$, uniformly in any compact subset of $E$.

For the sake of completeness, we give the proof of this result.

Proof of Lemma 5.1. By the Banach-Steinhaus theorem we get that $\left\|S_{n}\right\|$ is uniformly bounded with respect to $n$, and that $S$ is a bounded operator with $\|S\| \leq \liminf _{n}\left\|S_{n}\right\|$. 
Let $K$ be a compact subset of $E$ and let us assume that the sequence $S_{n}$ does not converge uniformly on $K$. Then, there exists a positive constant $\varepsilon_{0}>0$ and two sequences $\left(S_{n_{k}}\right)_{k \in \mathbb{N}}$ and $\left(x_{k}\right)_{k \in \mathbb{N}}, x_{k} \in K$, so that

$$
\left|S_{n_{k}} x_{k}-S x_{k}\right| \geq \varepsilon_{0} \quad \forall k \in \mathbb{N} .
$$

Thanks to the compactness of $K$, we can assume the sequence $\left(x_{k}\right)_{k \in \mathbb{N}}$ to converge to a limit $x, x \in K$. By writing

$$
S_{n_{k}} x_{k}-S x_{k}=S_{n_{k}} x_{k}-S_{n_{k}} x+S_{n_{k}} x-S x+S x-S x_{k},
$$

we get

$$
\varepsilon_{0} \leq\left|S_{n_{k}} x_{k}-S x_{k}\right| \leq 2 M\left\|x_{k}-x\right\|+\left\|S_{n_{k}} x-S x\right\|,
$$

where $M$ denotes $\sup _{n}\left\|S_{n}\right\|$.

Obviously this inequality contradicts the assumptions which imply the righthand side to converge to 0 .

5.1. Error estimate for regularizing the potential. In this subsection, the initial data $w_{I}$ is supposed to belong to $L^{2}\left(\mathbb{R}^{2}\right) \cap W^{m, \infty}\left(\mathbb{R}^{2}\right)$, with $m \geq 2$, and to satisfy $\left|\left\|w_{I} \mid\right\|_{m, \infty}<\infty\right.$.

We choose the mollifier

$$
\eta^{\alpha}(x)=\frac{1}{\alpha} \eta_{m}\left(\frac{x}{\alpha}\right), \quad x \in \mathbb{R},
$$

where $\eta_{m}$ is recursively defined by

$$
\eta_{k}=\eta_{0} * \eta_{k-1}, \quad 0<k \leq m,
$$

and

$$
\eta_{0}(x)= \begin{cases}1 & \text { if } x \in\left[-\frac{1}{2}, \frac{1}{2}\right] \\ 0 & \text { else }\end{cases}
$$

Then the Fourier transform of the regularized potential $V^{\alpha}=\eta^{\alpha} * V$ is equal to

$$
\widehat{V^{\alpha}}=\sqrt{2 \pi} \widehat{\eta^{\alpha}} \widehat{V}
$$

or explicitly

$$
\widehat{V^{\alpha}}(v)=\frac{1}{\sqrt{2 \pi}}\left[\frac{\sin (\alpha v / 2)}{(\alpha v / 2)}\right]^{m+1} \frac{\sin (v / 2)}{(v / 2)} \quad \forall v \in \mathbb{R} .
$$

This equality shows that the potential $V^{\alpha}$ satisfies Hypothesis 2. Therefore, the previous convergence analysis of the particle method holds for the corresponding Wigner equation,

$$
\left\{\begin{array}{l}
\partial_{t} w^{\alpha}+v \partial_{x} w^{\alpha}+E \partial_{v} w^{\alpha}=\varphi^{\alpha} *_{v} w^{\alpha} \\
w^{\alpha}(t=0)=w_{I}
\end{array}\right.
$$

where the function $\varphi^{\alpha}$ is given by (2.4):

$$
\varphi^{\alpha}(x, v)=\frac{2}{\pi} \operatorname{Im}\left[e^{2 i v x}\left(\frac{\sin (\alpha v)}{\alpha v}\right)^{m+1} \frac{\sin v}{v}\right] .
$$

The next proposition states that the solution $w^{\alpha}$ of equation (5.3) is a good approximation of the solution of equation (2.1) when $\alpha$ is small. 
Proposition 5.1. Let $T$ be a positive real number; then we have

$$
\sup _{t \in[0, T]}\left\|w(t)-w^{\alpha}(t)\right\|_{L^{2}\left(\mathbb{R}^{2}\right)} \rightarrow 0 \quad \text { when } \alpha \rightarrow 0 .
$$

Proof. Taking the difference between (2.1) and (5.3) gives

$$
\left\{\begin{array}{l}
\partial_{t}\left(w-w^{\alpha}\right)+v \partial_{x}\left(w-w^{\alpha}\right)+E \partial_{v}\left(w-w^{\alpha}\right) \\
=\left(\varphi-\varphi^{\alpha}\right) *_{v} w+\varphi^{\alpha} *_{v}\left(w-w^{\alpha}\right) \\
\left(w-w^{\alpha}\right)(t=0)=0
\end{array}\right.
$$

As a consequence, $\left(w-w^{\alpha}\right)$ is the solution of

$$
\left(w-w^{\alpha}\right)(t)=\int_{0}^{t} G_{t-s}\left[\varphi^{\alpha} *_{v}\left(w-w^{\alpha}\right)(s)+\left(\varphi-\varphi^{\alpha}\right) *_{v} w(s)\right] d s,
$$

where $G_{t}$ is the semigroup defined by (3.3).

This implies

$$
\begin{aligned}
\left\|\left(w-w^{\alpha}\right)(t)\right\|_{L^{2}\left(\mathbb{R}^{2}\right)} \leq \int_{0}^{t}[ & \left\|\varphi^{\alpha} *_{v}\left(w-w^{\alpha}\right)(s)\right\|_{L^{2}\left(\mathbb{R}^{2}\right)} \\
& \left.+\left\|\left(\varphi-\varphi^{\alpha}\right) *_{v} w(s)\right\|_{L^{2}\left(\mathbb{R}^{2}\right)}\right] d s .
\end{aligned}
$$

The second term of the integrand is bounded by

$$
\sup _{s \in[0, T]}\left\|\left(\varphi-\varphi^{\alpha}\right) *_{v} w(s)\right\|_{L^{2}\left(\mathbb{R}^{2}\right)}
$$

For the first term, we write the convolution product $\varphi^{\alpha} *_{v} u$, with $u \in L^{2}\left(\mathbb{R}^{2}\right)$, in its Fourier integral form:

$$
\varphi^{\alpha} *_{v} u=\frac{1}{2 \pi} \iint_{\mathbb{R}^{2}} \frac{1}{i}\left[V^{\alpha}\left(x-\frac{\eta}{2}\right)-V^{\alpha}\left(x+\frac{\eta}{2}\right)\right] u(x, \xi) e^{i(v-\xi) \eta} d \xi d \eta,
$$

which gives the estimates

$$
\begin{aligned}
\left\|\varphi^{\alpha} *_{v} u\right\|_{L^{2}\left(\mathbb{R}^{2}\right)} & \leq\left\|\left[V^{\alpha}\left(x-\frac{\eta}{2}\right)-V^{\alpha}\left(x+\frac{\eta}{2}\right)\right] \hat{u}(x, \eta)\right\|_{L^{2}\left(\mathbb{R}^{2}\right)} \\
& \leq 2\left\|V^{\alpha}\right\|_{L^{\infty}(\mathbb{R})}\|\hat{u}\|_{L^{2}\left(\mathbb{R}^{2}\right)}
\end{aligned}
$$

Since the norm $\left\|V^{\alpha}\right\|_{L^{\infty}(\mathbb{R})}$ is bounded by $\|V\|_{L^{\infty}(\mathbb{R})}$, estimate (5.5) implies

$$
\begin{aligned}
\left\|\left(w-w^{\alpha}\right)(t)\right\|_{L^{2}\left(\mathbb{R}^{2}\right)} \leq & T \sup _{s \in[0, T]}\left\|\left(\varphi-\varphi^{\alpha}\right) *_{v} w(s)\right\|_{L^{2}\left(\mathbb{R}^{2}\right)} \\
& +\int_{0}^{t} 2\|V\|_{L^{\infty}(\mathbb{R})}\left\|\left(w-w^{\alpha}\right)(s)\right\|_{L^{2}\left(\mathbb{R}^{2}\right)} d s \quad \forall t \in[0, T] .
\end{aligned}
$$

By Gronwall's lemma, we get existence of a constant $C(T)$, only depending on $T$, so that

$$
\left\|\left(w-w^{\alpha}\right)(t)\right\|_{L^{2}\left(\mathbb{R}^{2}\right)} \leq C(T) \sup _{s \in[0, T]}\left\|\left(\varphi-\varphi^{\alpha}\right) *_{v} w(s)\right\|_{L^{2}\left(\mathbb{R}^{2}\right)}
$$

holds for any $t \in[0, T]$.

It only remains to prove that the right-hand side of this estimate goes to 0 when $\alpha \rightarrow 0$. According to Lemma 5.1, and because $\{w(s), s \in[0, T]\}$ is a compact subset of $L^{2}\left(\mathbb{R}^{2}\right)$, this follows when it can be proven that the operator 
$\left(\varphi^{\alpha} *_{v}\right)$ converges to $\left(\varphi *_{v}\right)=\theta$ in the strong operator topology. Let $u$ belong to $L^{2}\left(\mathbb{R}^{2}\right)$. Then we have

$$
\begin{aligned}
& {\left[\left(\varphi^{\alpha}-\varphi\right) *_{v} u\right](x, v)} \\
& \quad=\frac{1}{2 \pi} \int \frac{1}{i}\left[\left(V^{\alpha}-V\right)\left(x-\frac{\eta}{2}\right)-\left(V^{\alpha}-V\right)\left(x+\frac{\eta}{2}\right)\right] u(x, \xi) e^{i(v-\xi) \eta} d \xi d \eta
\end{aligned}
$$

and

$$
\left\|\left(\varphi^{\alpha}-\varphi\right) *_{v} u\right\|_{L^{2}\left(\mathbb{R}^{2}\right)} \leq\left\|\left[\left(V^{\alpha}-V\right)\left(x-\frac{\eta}{2}\right)-\left(V^{\alpha}-V\right)\left(x+\frac{\eta}{2}\right)\right] \hat{u}(x, \eta)\right\|_{L^{2}\left(\mathbb{R}^{2}\right)} .
$$

Since the function $\eta_{m}$ which determines the mollifier $\eta^{\alpha}$ is compactly supported, we easily get

$$
\left|\left(V^{\alpha}-V\right)\left(x-\frac{\eta}{2}\right)-\left(V^{\alpha}-V\right)\left(x+\frac{\eta}{2}\right)\right| \rightarrow 0 \quad \text { when } \alpha \rightarrow 0
$$

almost everywhere in $\mathbb{R}_{x, \eta}^{2}$.

With

$$
\left|\left[\left(V^{\alpha}-V\right)\left(x-\frac{\eta}{2}\right)-\left(V^{\alpha}-V\right)\left(x+\frac{\eta}{2}\right)\right] \hat{u}(x, \eta)\right| \leq 4\|V\|_{L^{\infty}(\mathbb{R})}|\hat{u}(x, \eta)|,
$$

Lebesgue's theorem shows

$$
\left\|\left(\varphi^{\alpha}-\varphi\right) *_{v} u\right\|_{L^{2}\left(\mathbb{R}^{2}\right)} \rightarrow 0 \quad \text { when } \alpha \rightarrow 0,
$$

for any fixed $u \in L^{2}\left(\mathbb{R}^{2}\right)$.

Remark 5.1. In this proof we have never used the particular form of the potential $V$. Thus, Proposition 5.1 holds for any potential $V$ in $L^{\infty}(\mathbb{R})$.

The solution $w^{\alpha}$ of the regularized equation (5.3) can be approximated by the particle method. Because of the regularity of the potential $V^{\alpha}$, Theorem 4.1 applies in this case and provides an error estimate in $L^{\infty}\left(\mathbb{R}^{2}\right)$. Combined with the result of Proposition 5.1, it implies that this procedure furnishes an approximation $w_{h, \varepsilon}^{\alpha}$ converging to $w$ as $(\alpha, h / \varepsilon, \varepsilon) \rightarrow 0$.

5.2. A particle method preserving the discrete $l^{2}$-norm. Formally, we obtain such a particle method by replacing $(2.9 \mathrm{a})$ by

$$
\frac{d w_{i}}{d t}=\sum_{j \in \mathbb{Z}^{2}} \lambda_{j} \zeta^{\varepsilon}\left(x_{i}-x_{j}\right) \frac{\varphi\left(x_{i}, v_{i}-v_{j}\right)+\varphi\left(x_{j}, v_{i}-v_{j}\right)}{2} w_{j}, \quad i \in \mathbb{Z}^{2}
$$

This gives

$$
\sum_{i \in \mathbb{Z}^{2}} \lambda_{i} \frac{d w_{i}}{d t} w_{i}=\sum_{i, j \in \mathbb{Z}^{2}} \lambda_{i} \lambda_{j} \zeta^{\varepsilon}\left(x_{i}-x_{j}\right) \frac{\varphi\left(x_{i}, v_{i}-v_{j}\right)+\varphi\left(x_{j}, v_{i}-v_{j}\right)}{2} w_{j} w_{i}
$$

Because $\zeta^{\varepsilon}$ is even and $\varphi(x,-v)=-\varphi(x, v)$, the right-hand side is antisymmetric with respect to $(i, j)$, and therefore

$$
\frac{d}{d t}\left(\sum_{i} \lambda_{i} w_{i}^{2}\right)=0
$$

So, this scheme preserves the discrete $l^{2}$-norm provided all these expressions make sense, but it is no longer charge-conserving (cf. (2.10)). Indeed, we want to apply this method directly to the case of the rectangular potential barrier, 
without any mollifying. Here the convergence analysis is based on weak compactness methods following the ideas of [15]. It relies on a weak formulation of a regularized Wigner equation with the right-hand side $\theta^{\varepsilon} w$, as defined in $(2.8)$. The weak convergence result will then be improved to obtain strong convergence towards the classical solution of the Wigner equation $[15,16]$.

Here, the existence of a solution for the differential system (5.6) is questionable, because the stability analysis of $\S 4.2$ (relying on Hypothesis 2 ) no longer holds. We will overcome this problem by considering only a finite number of particles. This number of particles is related to the discretization parameter $h$ in the following way: We introduce the domain $D_{h}=\left[-X_{h}, X_{h}\right] \times\left[-V_{h}, V_{h}\right]$ in $\mathbb{R}^{2}$ such that

$$
X_{h} \rightarrow \infty \text { and } V_{h} \rightarrow \infty \text { when } h \rightarrow 0,
$$

and we define the set $I_{h}$ of indices by

$$
I_{h}=\left\{(l, k) \in \mathbb{Z}^{2} \mid(l h, k h) \in D_{h}\right\} .
$$

By denoting $i=(l, k)$ and $K_{i}=\left[\left(l-\frac{1}{2}\right) h,\left(l+\frac{1}{2}\right) h\right] \times\left[\left(k-\frac{1}{2}\right) h,\left(k+\frac{1}{2}\right) h\right]$, the differential system for the weights $w_{i}(t), i \in I_{h}$, reads

$$
\left\{\begin{array}{l}
\frac{d w_{i}}{d t}=\sum_{j \in I_{h}} h^{2} \zeta^{\varepsilon}\left(x_{i}-x_{j}\right) \frac{\varphi\left(x_{i}, v_{i}-v_{j}\right)+\varphi\left(x_{j}, v_{i}-v_{j}\right)}{2} w_{j}, \\
w_{i}(0)=\frac{1}{h} \sqrt{\int_{K_{i}}\left|w_{I}(x, v)\right|^{2} d x d v}
\end{array}\right.
$$

First, we will analyze the approximation property of the solution of (5.7) for a fixed $\varepsilon$ and $h \rightarrow 0$. As we have seen above, the solution of this system preserves its $l^{2}$-norm:

$$
\sum_{i \in I_{h}} h^{2} w_{i}(t)^{2}=\sum_{i \in I_{h}} h^{2} w_{i}(0)^{2}=\int_{D_{h}} w_{I}(x, v)^{2} d x d v .
$$

Next we also introduce the function $X_{i}(x, v, t)=G_{t}\left[1_{K_{i}}(x, v)\right]$, which satisfies

$$
\left\{\begin{array}{l}
\partial_{t} X_{i}+v \partial_{x} X_{i}+E \partial_{v} X_{i}=0, \\
X_{i}(x, v, 0)= \begin{cases}1 & \text { if }(x, v) \in K_{i}, \\
0 & \text { else. }\end{cases}
\end{array}\right.
$$

Then we construct the time-dependent function $w^{h}(t) \in L^{2}\left(\mathbb{R}^{2}\right)$ by interpolation:

$$
w^{h}(x, v, t)=\sum_{i \in I_{h}} w_{i}(t) X_{i}(x, v, t)
$$

Its $L^{2}$-norm is given by

$$
\left\|w^{h}(t)\right\|_{L^{2}\left(\mathbb{R}^{2}\right)}=\sqrt{\sum_{i \in I_{h}} h^{2} w_{i}(t)^{2}},
$$

and because of the conservativity property of (5.7), we get

$$
\left\|w^{h}(t)\right\|_{L^{2}\left(\mathbb{R}^{2}\right)}=\left\|w_{I}\right\|_{L^{2}\left(D_{h}\right)} \leq\left\|w_{I}\right\|_{L^{2}\left(\mathbb{R}^{2}\right)} .
$$


Considering $w^{h} \in L^{2}\left((0, T) \times \mathbb{R}^{2}\right)$ gives

$$
\left\|w^{h}\right\|_{L^{2}\left((0, T) \times \mathbb{R}^{2}\right)}=\sqrt{T}\left\|w_{I}\right\|_{L^{2}\left(D_{h}\right)} \leq \sqrt{T}\left\|w_{I}\right\|_{L^{2}\left(\mathbb{R}^{2}\right)} .
$$

As a consequence, we can extract a subsequence, still denoted by $w^{h}$, such that $w^{h}-w^{0}$ in $L^{2}\left((0, T) \times \mathbb{R}^{2}\right)$ weakly as $h \rightarrow 0$.

Lemma 5.2. The function $w^{0} \in L^{2}\left((0, T) \times \mathbb{R}^{2}\right)$ is a weak solution of the equation

$$
\left\{\begin{array}{l}
\partial_{t} w^{0}+v \partial_{x} w^{0}+E \partial_{v} w^{0}=\tilde{\theta}^{\varepsilon} w^{0} \\
w^{0}(t=0)=w_{I}
\end{array}\right.
$$

where the operator $\tilde{\theta}^{\varepsilon}$ is defined by

$$
\tilde{\theta}^{\varepsilon} u=\frac{1}{2}\left[\theta^{\varepsilon} u+\theta\left(\zeta^{\varepsilon} *_{x} u\right)\right]=\frac{1}{2}\left[\zeta^{\varepsilon} *_{x}(\theta u)+\theta\left(\zeta^{\varepsilon} *_{x} u\right)\right] \quad \forall u \in L^{2}\left(\mathbb{R}^{2}\right) .
$$

Here "weak solution" means an element of $\mathscr{D}^{\prime}\left([0, T) \times \mathbb{R}^{2}\right)$ which satisfies

$$
\begin{gathered}
\int_{0}^{T} \int_{\mathbb{R}^{2}} w^{0}\left(-\partial_{t} \psi-v \partial_{x} \psi-E \partial_{v} \psi\right) d x d v d t+\int_{\mathbb{R}^{2}} w_{I} \psi(t=0) d x d v \\
=-\int_{0}^{T} \int_{\mathbb{R}^{2}} w^{0}\left(\tilde{\theta}^{\varepsilon} \psi\right) d x d v d t \quad \forall \psi \in \mathscr{D}\left([0, T) \times \mathbb{R}^{2}\right) .
\end{gathered}
$$

Proof. Let $\psi$ belong to $\mathscr{D}\left([0, T) \times \mathbb{R}^{2}\right)$. For any $i \in I_{h}$, we set

$$
\psi_{i}(t)=\psi\left(x_{i}(t), v_{i}(t), t\right), \quad t \in[0, T),
$$

and we have

$$
\frac{d \psi_{i}}{d t}(t)=\left[\left(\partial_{t}+v \partial_{x}+E \partial_{v}\right) \psi\right]\left(x_{i}(t), v_{i}(t), t\right) \quad \forall i \in I_{h}, \forall t \in[0, T) .
$$

As we did for $w^{h}$ in (5.8), we now define the interpolation functions $\psi^{h}, \Psi^{h}$, and $f^{h}$ :

$$
\begin{gathered}
\psi^{h}(x, v)=\sum_{i \in I_{h}} \psi_{i}(0) X_{i}(x, v, 0), \\
\Psi^{h}(x, v, t)=\sum_{i \in I_{h}}\left(\frac{d \psi_{i}}{d t}\right)(t) X_{i}(x, v, t), \\
f^{h}(x, v, t)=\sum_{i \in I_{h}} f_{i}(t) X_{i}(x, v, t),
\end{gathered}
$$

where the functions $f_{i}(t)$ are given by

$$
f_{i}(t)=\sum_{j \in I_{h}} h^{2} \zeta^{\varepsilon}\left(x_{j}-x_{i}\right) \frac{\varphi\left(x_{i}, v_{j}-v_{i}\right)+\varphi\left(x_{j}, v_{j}-v_{i}\right)}{2} \psi_{j}(t) .
$$

We multiply equation (5.7) by $h^{2} \psi_{i}(t)$ and sum up:

$$
\begin{aligned}
\sum_{i \in I_{h}} h^{2} \frac{d w_{i}}{d t} \psi_{i} & =\sum_{i, j \in I_{h}} h^{4} \zeta^{\varepsilon}\left(x_{i}-x_{j}\right) \frac{\varphi\left(x_{i}, v_{i}-v_{j}\right)+\varphi\left(x_{j}, v_{i}-v_{j}\right)}{2} w_{j} \psi_{i} \\
& =\sum_{j \in I_{h}} h^{2} w_{j}\left[\sum_{i \in I_{h}} h^{2} \zeta^{\varepsilon}\left(x_{i}-x_{j}\right) \frac{\varphi\left(x_{i}, v_{i}-v_{j}\right)+\varphi\left(x_{j}, v_{i}-v_{j}\right)}{2} \psi_{i}\right] \\
& =\sum_{j \in I_{h}} h^{2} w_{j} f_{j} .
\end{aligned}
$$


Integrating with respect to $t$ gives

$$
\begin{aligned}
\int_{0}^{T} & {\left[\sum_{i \in I_{h}} h^{2} w_{i}(t)\left(-\frac{d \psi_{i}}{d t}\right)(t)\right] d t+\sum_{i \in I_{h}} h^{2} w_{i}(0) \psi_{i}(0) } \\
& =\int_{0}^{T}\left[\sum_{j \in I_{h}} h^{2} w_{j}(t) f_{j}(t)\right] d t
\end{aligned}
$$

and by introducing $\psi^{h}, \Psi^{h}$, and $f^{h}$, we obtain

$$
\begin{gathered}
\int_{0}^{T} \int_{\mathbb{R}^{2}} w^{h}(x, v, t)\left(-\Psi^{h}(x, v, t)\right) d x d v d t \\
\quad+\int_{\mathbb{R}^{2}} w^{h}(x, v, 0) \psi^{h}(x, v) d x d v \\
\quad=\int_{0}^{T} \int_{\mathbb{R}^{2}} w^{h}(x, v, t) f^{h}(x, v, t) d x d v d t .
\end{gathered}
$$

Since the test function $\psi$ belongs to $\mathscr{D}\left([0, T) \times \mathbb{R}^{2}\right)$, we easily get

$$
\begin{array}{ll}
\psi^{h} \rightarrow \psi(t=0) & \text { in } L^{2}\left(\mathbb{R}^{2}\right), \\
\Psi^{h} \rightarrow\left(\partial_{t}+v \partial_{x}+E \partial_{v}\right) \psi & \text { in } L^{2}\left((0, T) \times \mathbb{R}^{2}\right), \text { as } h \rightarrow 0 .
\end{array}
$$

Moreover, we already know that

$$
\begin{array}{ll}
w^{h}(t=0) \rightarrow w_{I} & \text { in } L^{2}\left(\mathbb{R}^{2}\right) \\
w^{h} \rightarrow w^{0} & \text { in } L^{2}\left(\mathbb{R}^{2} \times(0, T)\right) \text { weak. }
\end{array}
$$

Then equation (5.10) is obtained by taking the limit of the previous equality as $h \rightarrow 0$, as soon as we have proved strong convergence:

$$
f^{h} \rightarrow\left(-\widetilde{\theta}^{\varepsilon} \psi\right) \text { in } L^{2}\left(\mathbb{R}^{2} \times(0, T)\right), \text { as } h \rightarrow 0 .
$$

Hereafter, $F$ will denote the function defined by

$$
F\left(x, v, x^{\prime}, v^{\prime}\right)=\zeta^{\varepsilon}\left(x^{\prime}-x\right) \frac{\varphi\left(x^{\prime}, v^{\prime}-v\right)+\varphi\left(x, v^{\prime}-v\right)}{2},
$$

$\left(x, v, x^{\prime}, v^{\prime}\right) \in \mathbb{R}^{4}$, where we recall

$$
\varphi(x, v)=\frac{2}{\pi} \operatorname{Im}\left[e^{2 i v x} \frac{\sin v}{v}\right] .
$$

Because of the regularity of $\zeta^{\varepsilon}$ (Hypothesis 1$), F$ is a continuous function, and there are constants $C$ and $D$ such that

$$
F\left(x, v, x^{\prime}, v^{\prime}\right) \leq \frac{C}{1+\left|v-v^{\prime}\right|}
$$

and

$$
F\left(x, v, x^{\prime}, v^{\prime}\right)=0 \quad \text { if }\left|x-x^{\prime}\right| \geq D
$$


Since $\psi$ belongs to $\mathscr{D}\left([0, T) \times \mathbb{R}^{2}\right)$ and $F$ is continuous, the sum

$$
\sum_{i \in I_{h}} h^{2} F\left(x, v, x_{i}, v_{i}\right) \psi\left(x_{i}, v_{i}, t\right)
$$

converges to

$$
\int_{\mathbb{R}^{2}} F\left(x, v, x^{\prime}, v^{\prime}\right) \psi\left(x^{\prime}, v^{\prime}, t\right) d x^{\prime} d v^{\prime}=-\left(\tilde{\theta}^{\varepsilon} \psi\right)(x, v, t)
$$

as $h \rightarrow 0$, for any fixed $(x, v, t) \in \mathbb{R}^{2} \times(0, T)$. We fix $(x, v, t) \in \mathbb{R}^{2} \times(0, T)$ and assume that $h$ is small enough in order to ensure $\sum_{j \in I_{h}} X_{j}(x, v, t)=1$.

Then, we have

$$
\begin{gathered}
\left|f^{h}(x, v, t)-\sum_{i \in I_{h}} h^{2} F\left(x, v, x_{i}, v_{i}\right) \psi\left(x_{i}, v_{i}, t\right)\right| \\
=\mid \sum_{j \in I_{h}} X_{j}(x, v, t)\left[\sum _ { i \in I _ { h } } h ^ { 2 } \left(F\left(x_{j}, v_{j}, x_{i}, v_{i}\right)\right.\right. \\
\left.\left.-F\left(x, v, x_{i}, v_{i}\right)\right)\right] \psi\left(x_{i}, v_{i}, t\right) \mid,
\end{gathered}
$$

and the right-hand side can be estimated by

$$
\left[\sum_{i \in I_{h}} h^{2}\left|\psi\left(x_{i}, v_{i}, t\right)\right|\right] \times \sup _{\substack{\left(x^{\prime}, v^{\prime}\right) \in \operatorname{supp} \psi(t) \\\left|x^{\prime \prime}-x\right| \leq h \\\left|v^{\prime \prime}-v\right| \leq h}}\left(F\left(x^{\prime \prime}, v^{\prime \prime}, x^{\prime}, v^{\prime}\right)-F\left(x, v, x^{\prime}, v^{\prime}\right)\right) \text {. }
$$

The first factor is bounded by a constant and the second converges to 0 as $h \rightarrow 0$, because $F$ is uniformly continuous on the compact set $\left\{\left(x^{\prime \prime}, v^{\prime \prime}\right) \in\right.$ $\mathbb{R}^{2}|| x^{\prime \prime}-x \mid \leq 1$ and $\left.\left|v^{\prime \prime}-v\right| \leq 1\right\} \times \operatorname{supp} \psi(t)$. This proves that for any $(x, v, t) \in \mathbb{R}^{2} \times(0, T), f^{h}(x, v, t)$ converges to $\left(-\tilde{\theta}^{\varepsilon} \psi\right)(x, v, t)$ as $h \rightarrow 0$. Moreover, the decay properties of $F,(5.11)$ and (5.12), and the compactness of supp $\psi(t)$ provide two constants $C^{\prime}$ and $D^{\prime}$ (independent of $h$ ) such that

$$
\forall(x, v, t) \in \mathbb{R}^{2} \times(0, T)\left\{\begin{array}{l}
f^{h}(x, v, t) \leq \frac{C^{\prime}}{1+|v|} \text { and }\left|\tilde{\theta}^{\varepsilon} \psi(x, v, t)\right| \leq \frac{C^{\prime}}{1+|v|}, \\
f^{h}(x, v, t)=\left(\tilde{\theta}^{\varepsilon} \psi\right)(x, v, t)=0 \text { if }|x| \geq D^{\prime}
\end{array}\right.
$$

Then, by applying Lebesgue's theorem to the function $\left|f^{h}+\tilde{\theta}^{\varepsilon} \psi\right|^{2}$, we obtain the convergence

$$
f^{h} \rightarrow-\tilde{\theta}^{\varepsilon} \psi \text { in } L^{2}\left(\mathbb{R}^{2} \times(0, T)\right) .
$$

The above convergence result can now be improved.

Proposition 5.2. There exists a unique solution $w^{\varepsilon}$ in $C\left([0, T), L^{2}\left(\mathbb{R}^{2}\right)\right)$ of equation (5.9), and the whole sequence $w^{h}$ converges strongly to $w^{\varepsilon}$ in $L^{2}\left(\mathbb{R}^{2} \times(0, T)\right)$, as $h \rightarrow 0$.

Proof. From the estimate

$$
\left\|\zeta^{\varepsilon} *_{x} u\right\|_{L^{2}\left(\mathbb{R}^{2}\right)} \leq\|u\|_{L^{2}\left(\mathbb{R}^{2}\right)}
$$


and the boundedness of the operator $\theta$ in $L^{2}\left(\mathbb{R}^{2}\right)$, we conclude that $\tilde{\theta}^{\varepsilon}$ is bounded. Since this operator satisfies

$$
\int_{\mathbb{R}^{2}} u\left(\tilde{\theta}^{\varepsilon} u\right) d x d v=0 \quad \forall u \in L^{2}\left(\mathbb{R}^{2}\right)
$$

there exists a unique mild solution of equation (5.9), $w^{\varepsilon} \in C\left([0, T), L^{2}\left(\mathbb{R}^{2}\right)\right)$, with

$$
\left\|w^{\varepsilon}(t)\right\|_{L^{2}\left(\mathbb{R}^{2}\right)}=\left\|w_{I}\right\|_{L^{2}\left(\mathbb{R}^{2}\right)} \quad \forall t \in[0, T) .
$$

We denote $e=w^{0}-w^{\varepsilon}$, where $w^{0}$ is the function introduced in Lemma 5.2 as the limit of a subsequence of $\left(w^{h}\right)$. This function, belonging to $L^{2}\left(\mathbb{R}^{2} \times(0, T)\right)$, is a weak solution of

$$
\left\{\begin{array}{l}
\partial_{t} e+v \partial_{x} e+E \partial_{v} e=\tilde{\theta}^{\varepsilon} e \\
e(t=0)=0
\end{array}\right.
$$

Therefore, we have

$$
\partial_{t} e+v \partial_{x} e+E \partial_{v} e \in L^{2}\left(\mathbb{R}^{2} \times(0, T)\right),
$$

and for almost every $t \in(0, T)$

$$
\int_{\mathbb{R}^{2}}\left(\partial_{t} e+v \partial_{x} e+E \partial_{v} e\right) e d x d v=0
$$

Using the unitary group $G_{t}$, we see that this is equivalent to

$$
\frac{d}{d t}\left\|G_{-t} e(t)\right\|_{L^{2}\left(\mathbb{R}^{2}\right)}=0
$$

which leads to

$$
\|e(t)\|_{L^{2}\left(\mathbb{R}^{2}\right)}=\left\|G_{-t} e(t)\right\|_{L^{2}\left(\mathbb{R}^{2}\right)}=0 \text { for almost every } t \in(0, T) .
$$

Thus, we have $\|e\|_{L^{2}\left((0, T) \times \mathbb{R}^{2}\right)}=0$ and $w^{0}=w^{\varepsilon}$. The uniqueness of the limit $w^{0}$ implies that the whole sequence $\left(w^{h}\right)$ converges to $w^{0}=w^{\varepsilon}$,

$$
w^{h} \rightarrow w^{\varepsilon} \text { in } L^{2}\left(\mathbb{R}^{2} \times(0, T)\right) \text { weak. }
$$

Moreover, the convergence of the norm

$$
\left\|w^{h}\right\|_{L^{2}\left(\mathbb{R}^{2} \times(0, T)\right)}=\sqrt{T}\left\|w^{h}(t=0)\right\|_{L^{2}\left(\mathbb{R}^{2}\right)}
$$

to

$$
\sqrt{T}\left\|w_{I}\right\|_{L^{2}\left(\mathbb{R}^{2}\right)}=\left\|w^{\varepsilon}\right\|_{L^{2}\left(\mathbb{R}^{2} \times(0, T)\right)}
$$

implies

$$
w^{h} \rightarrow w^{\varepsilon} \quad \text { in } L^{2}\left(\mathbb{R}^{2} \times(0, T)\right) \text { strong. }
$$

The cutoff function $\zeta^{\varepsilon}$, as an approximation of the delta function, satisfies

$$
\left\|u-\zeta^{\varepsilon} *_{x} u\right\|_{L^{2}\left(\mathbb{R}^{2}\right)} \rightarrow 0 \text { for } \varepsilon \rightarrow 0, u \in L^{2}\left(\mathbb{R}^{2}\right) .
$$

As a consequence, it is easily proved that $\tilde{\theta}^{\varepsilon}$ converges to $\theta$ in the strong operator topology. Therefore, we can apply the same analysis as in $\S 5.1$ and state the final result in 
Theorem 5.1. For any fixed $\varepsilon$, the sequence $w^{h}$ defined by (5.8) satisfies

$$
w^{h} \rightarrow w^{\varepsilon} \quad \text { in } L^{2}\left(\mathbb{R}^{2} \times(0, T)\right)
$$

and further, as $\varepsilon \rightarrow 0$, we have

$$
\sup _{t \in[0, T]}\left\|w^{\varepsilon}(t)-w(t)\right\|_{L^{2}\left(\mathbb{R}^{2}\right)} \rightarrow 0
$$

\section{ACKNOWLEDGMENT}

This work was carried out while the first author visited the "Centre de Mathématiques Appliquées de l'Ecole Polytechnique." He was also supported by the "Austrian Fonds zur Förderung der Wissenschaftlichen Forschung," grant No. P6771.

\section{BIBLIOGRAPHY}

1. S. Mas-Gallic and P. A. Raviart, A particle method for first-order symmetric systems, Numer. Math. 51 (1987), 323-352.

2. E. P. Wigner, On the quantum correction for thermodynamic equilibrium, Phys. Rev. 40 (1932), 749-759.

3. W. R. Frensley, Wigner function model of a resonant-tunneling semiconductor device, Phys. Rev. B 36 (1987), 1570-1580.

4. U. Ravaioli, M. A. Osman, W. Pötz, N. Kluksdahl, and D. K. Ferry, Investigation of ballistic transport through resonant-tunneling quantum wells using Wigner function approach, Physica B 134 (1985), 36-40.

5. C. Ringhofer, A spectral method for the numerical simulation of quantum tunneling phenomena, SIAM J. Numer. Anal. 27 (1991), 32-50.

6. S. Mas-Gallic and F. Poupaud, Approximation of the transport equation by a particle method, Trans. Theory Statist. Phys. 17 (1988), 311-345.

7. S. Mas-Gallic, A deterministic particle method for the linearized Boltzmann equation, Trans. Theory Statist. Phys. 16 (1987), 855-857.

8. P. Degond and B. Niclot, Numerical analysis of the weighted particle method applied to the semiconductor Boltzmann equation, Numer. Math. 55 (1989), 599-618.

9. P. A. Markowich, On the equivalence of the Schrödinger and the quantum Liouville equations, Math. Meth. Appl. Sci. 11 (1989), 459-469.

10. P. A. Raviart, An analysis of particle methods, Lecture Notes in Math., vol. 1127, SpringerVerlag, Berlin, Heidelberg, New York, 1985.

11. Robert A. Adams, Sobolev spaces, Academic Press, New York, 1975.

12. P. Degond and F. Guyot-Delaurens, Particle simulations of the semiconductor Boltzmann equation for one-dimensional homogeneous structures, J. Comput. Phys. (submitted).

13. A. Pazy, Semigroups of linear operators and applications to partial differential equations, Springer-Verlag, 1983.

14. G. H. Cottet and P. A. Raviart, Particle methods for the one-dimensional Vlasov-Poisson equations, SIAM J. Numer. Anal. 21 (1984), 52-76.

15. P. Degond and P. A. Markowich, $A$ mathematical analysis of quantum transport in threedimensional crystals, Ann. Mat. Pura Appl. (to appear).

16. __ A quantum transport model for semiconductors: the Wigner-Poisson problem on a bounded Brillouin zone, Math. Mod. Numer. Anal. (to appear).

17. R. Dautray and J. L. Lions, Analyse mathématique et calcul numérique, Masson, Paris, 1985. 
18. P. A. Markowich, C. Ringhofer, and C. Schmeiser, Semiconductor equations, SpringerVerlag, Wien-New York, 1990.

19. F. Nier, Application de la méthode particulaire à l'équation de Wigner-mise en oeuvre numérique, Thèse de l'Ecole Polytechnique, Palaiseau, France, 1991.

20. M. Reed and B. Simon, Methods of modern mathematical physics II: Fourier analysis, selfadjointness, Academic Press, New York, 1972.

(A. Arnold) Department of Mathematics, Purdue University, West Lafayette, Indiana 47907

(A. Arnold) Fachbereich Mathematik, TU-Berlin, Strasse des 17 Juni 136, D-1000 Berlin 12, GeRMANY

(F. Nier) Centre de Mathématiques Appliquées, Ecole Polytechnique, F-91128 Palaiseau Cedex, France 